\title{
Symmetric elastic knots
}

\author{
Alexandra Gilsbach ${ }^{1}$ (D) Philipp Reiter ${ }^{2}$ (D) $\cdot$ Heiko von der Mosel $^{3}$ (D)
}

Received: 23 August 2021 / Revised: 6 December 2021 / Accepted: 9 December 2021 /

Published online: 31 January 2022

(c) The Author(s) 2022

\begin{abstract}
Minimizing the bending energy within knot classes leads to the concept of elastic knots which has been initiated by von der Mosel (Asymptot Anal 18(1-2):49-65, 1998). Motivated by numerical experiments in Bartels and Reiter (Math Comput 90(330):1499-1526, 2021) we prescribe dihedral symmetry and establish existence of dihedrally symmetric elastic knots for knot classes admitting this type of symmetry. Among other results we prove that the dihedral elastic trefoil is the union of two circles that form a (planar) figure-eight. We also discuss some generalizations and limitations regarding other symmetries and knot classes.
\end{abstract}

\section{Contents}

1 Introduction . . . . . . . . . . . . . . . . . . . . . . . . 812

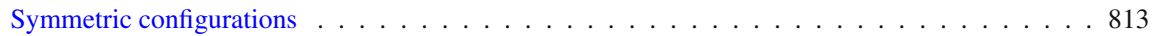

Existence results . . . . . . . . . . . . . . . . . . . . . . . . . . . . 814

Shapes of symmetric elastic knots . . . . . . . . . . . . . . . . . . . . 815

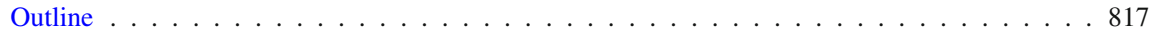

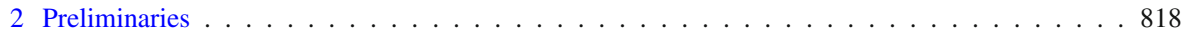

2.1 Principle of symmetric criticality . . . . . . . . . . . . . . . . . . . . . 818

2.2 Tangent-point energy . . . . . . . . . . . . . . . . . . . . . . . 819

Communicated by Giga.

$凶 \quad$ Philipp Reiter

reiter@math.tu-chemnitz.de

Alexandra Gilsbach

gilsbach.a.aa@m.titech.ac.jp

Heiko von der Mosel

heiko@instmath.rwth-aachen.de

1 Department of Mathematics, School of Science, Tokyo Institute of Technology, 2-12-1

Ookayama, Meguro-ku, Tokyo 152-8551, Japan

2 Chemnitz University of Technology, Faculty of Mathematics, 09107 Chemnitz, Germany

3 RWTH Aachen University, Institut für Mathematik, Templergraben 55, 52062 Aachen, Germany 
2.3 Isotopy stability . . . . . . . . . . . . . . . . . . . . . . . . . . . 820

3 Group action on parametrized curves . . . . . . . . . . . . . . . . . . . . . 820

4 Existence theory under the $D_{2}$-symmetry constraint $\ldots \ldots \ldots \ldots$. . . . . . . . . . . 832

5 Infimal bending energy on torus knots with dihedral symmetry . . . . . . . . . . . . . . . 838

6 Discussion and open problems . . . . . . . . . . . . . . . . . . . . . . . . . . . . . . . 840

6.1 Higher regularity of $D_{2}$-symmetric $E_{\vartheta}$-critical knots $\ldots \ldots \ldots \ldots$. . . . . . . . . . 840

6.2 Non-embeddedness of symmetric elastic knots . . . . . . . . . . . . . . . . . . . . . . 840

6.3 Other knot classes and symmetries . . . . . . . . . . . . . . . . . . . . . 841

General torus knots . . . . . . . . . . . . . . . . . . . . . . . 841

The figure-eight knot . . . . . . . . . . . . . . . . . . . . . . . . 842

References . . . . . . . . . . . . . . . . . . . . . . . . . . . 842

\section{Introduction}

The study of elastic knots was initiated by Gerlach et al. in [24]. Inspired by toy models of springy knotted wires (see the images in [24, Figure 7]) the existence of energy minimizing knotted configurations $\gamma_{\vartheta}$ has been established in any prescribed tame ${ }^{1}$ knot class $\mathcal{K}$ [24, Theorem 2.1]. The total energy considered,

$$
E_{\vartheta}:=E+\vartheta \mathcal{R}, \quad \text { for } \vartheta>0
$$

consists of the classic Euler-Bernoulli bending energy

$$
E(\gamma):=\int_{\gamma} \kappa^{2} d s
$$

as the leading order term, together with a small multiple of a repulsive potential $\mathcal{R}$ to avoid self-intersections. In order to analyse the approximative shape of the minimizing knots $\gamma_{\vartheta}$ for small $\vartheta$ the authors study the limit $\vartheta \rightarrow 0$. It is shown that the minimizers $\gamma_{\vartheta}$ converge in $C^{1}$ to closed curves $\gamma_{0}$ that minimize the bending energy

$$
E\left(\gamma_{0}\right) \leq E(\beta) \quad \text { for all } \beta \in \mathscr{C}(\mathcal{K})
$$

where

$$
\mathscr{C}(\mathcal{K}):=\left\{\gamma \in W^{2,2}\left(\mathbb{R} / \mathbb{Z}, \mathbb{R}^{3}\right): \mathscr{L}(\gamma)=1,\left|\gamma^{\prime}\right|>0,[\gamma]=\mathcal{K}\right\}
$$

Here, $\mathbb{R} / \mathbb{Z}$ denotes the periodic interval of unit length. These limiting curves $\gamma_{0}$ are called elastic knots for $\mathcal{K}$ according to [24, Definition 2.3], although they are not embedded unless $\mathcal{K}$ is trivial; see [24, Proposition 3.1]. One of the central results is the complete classification of elastic knots for all torus knot classes $\mathcal{T}(2, b)$ for odd $b \in \mathbb{Z} \backslash\{1,-1\}$.

Theorem ([24, Corollary 6.5(i)]) The elastic torus knot $\gamma_{0}$ for $\mathcal{T}(2, b)$ for any odd $b \in \mathbb{Z} \backslash\{1,-1\}$ is the doubly covered circle.

\footnotetext{
1 A knot class is called tame if it contains a polygonal representative [14, Definition 1.3], or equivalently, if and only if it contains a continuously differentiable representative [17, App. I].
} 

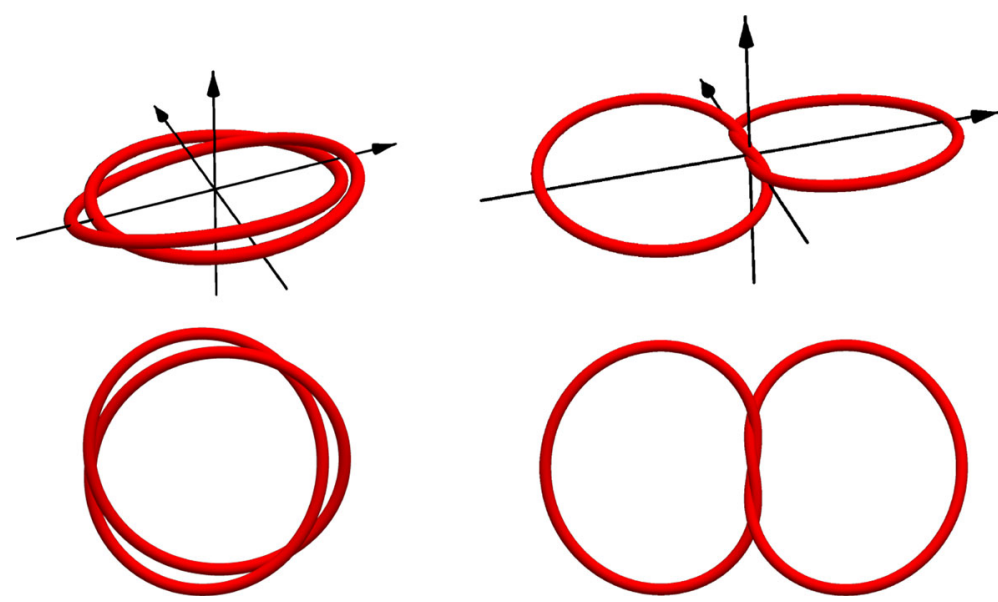

Fig. 1 Final states of the numerical gradient flow of Bartels et al. [7] for the total energy $E_{\vartheta}$ for small $\vartheta>0$ in the case of the trefoil knot class. Left: The doubly covered circle is the usual limit configuration as predicted by [24]. Right: There are a few initial configurations that move towards an almost flat torus knot with dihedral symmetry under the gradient flow

This result is confirmed by mechanical experiments with thin elastic knotted wires, as well as by various numerical simulations performed by different groups of researchers as documented in [24, Introduction p. 94]. Also the more recent work on the corresponding numerical gradient flow of Bartels et al. [6,7] provides strong numerical evidence for the doubly covered circle as the only possible elastic knot for $\mathcal{T}(2, b)$; see the left of Fig. 1.

\section{Symmetric configurations}

Sometimes, however, this numerical gradient flow produces a different limiting configuration exhibiting a dihedral symmetry as depicted on the right of Fig. 1. This indicates the presence of a dihedrally symmetric critical point ${ }^{2}$ of the total energy $E_{\vartheta}$.

It is the purpose of the present paper to analytically support these infrequent but reproducible numerical observations. Namely, we use the principle of symmetric criticality of Palais [33] to prove the existence of symmetric critical points for the constrained variational problem

Minimize the total energy $E_{\vartheta}$ on the set $\mathscr{C}(\mathcal{K})$.

$\left(\mathrm{P}_{\vartheta}\right)$

Palais's principle, however, requires energy functionals of class $C^{1}$. To meet this precondition (and to avoid the technical issues connected with an alternative nonsmooth

\footnotetext{
2 This symmetric knot might be a saddle point, since there exist—as reported in [6]—-symmetry breaking perturbations with smaller energy. A more systematic numerical investigation is under way to produce more evidence of the nature of this critical point.
} 
variant [15] of this principle) we replace the nonsmooth ropelength functional used in [24] by a suitable power of the tangent-point energy $\mathrm{TP}_{q}$. It is a self-avoiding energy defined on absolutely continuous regular closed curves $\gamma: \mathbb{R} / \mathbb{Z} \rightarrow \mathbb{R}$ as

$$
\mathcal{R}(\gamma):=\operatorname{TP}_{q}^{\frac{1}{q-2}}(\gamma):=\left(\int_{\mathbb{R} / \mathbb{Z}} \int_{\mathbb{R} / \mathbb{Z}} \frac{1}{r_{\mathrm{tp}}^{q}(\gamma(s), \gamma(t))}\left|\gamma^{\prime}(s)\right|\left|\gamma^{\prime}(t)\right| d s d t\right)^{\frac{1}{q-2}}
$$

where we restrict to exponents $q \in(2,4]$. Here, $r_{\operatorname{tp}}(\gamma(s), \gamma(t))$ stands for the radius of the unique circle through the points $\gamma(s)$ and $\gamma(t)$ that is tangent to $\gamma$ at $\gamma(s)$. This energy was first suggested by Buck and Orloff [13] in the case $q=2$ and for general $q>2$ by Gonzalez and Maddocks in [27, p. 4773], and it was investigated analytically in detail in [38], [9], and [10]. Note that the Sobolev space $W^{2,2}$ continuously embeds into the fractional Sobolev space $W^{2-(1 / q), q}$ for $q \in(2,4]$, which provides the exact regularity framework to guarantee a finite and continuously differentiable tangent point energy [10, Remark 3.1], [42], so that the total energy $E_{\vartheta}$ is continuously differentiable on the open subset $W_{\text {ir }}^{2,2}\left(\mathbb{R} / \mathbb{Z}, \mathbb{R}^{3}\right)$ of injective regular closed curves of class $W^{2,2}$. Consequently, Palais's principle of symmetric criticality is applicable. Furthermore, a suitably discretized version of $\mathrm{TP}_{q}$ for $q \in(2,4]$ was used for $\mathcal{R}$ in the numerical gradient flow in [7] and [6].

\section{Existence results}

Theorem 1.1 (Existence of symmetric critical knots) Given a knot class $\mathcal{K}$, assume that there is at least one knot with dihedral symmetry contained in $\mathscr{C}(\mathcal{K})$. Then for every $\vartheta>0$ there exists an arclength parametrized knot $\Gamma_{\vartheta}$ of knot type $\mathcal{K}$ with dihedral symmetry that is critical for the constrained minimization problem $\left(P_{\vartheta}\right)$. More precisely, we have

$$
D E_{\vartheta}\left(\Gamma_{\vartheta}\right) h+\lambda D \mathscr{L}\left(\Gamma_{\vartheta}\right) h=0 \quad \text { for all } h \in W^{2,2}\left(\mathbb{R} / \mathbb{Z}, \mathbb{R}^{3}\right),
$$

where $\lambda:=E_{\vartheta}\left(\Gamma_{\vartheta}\right)$.

Here, the term dihedral symmetry ${ }^{3}$ or, synonymously, $D_{2}$-symmetry refers to the action of the classic dihedral group $D_{2}$ (see Table 1) on parametrized space curves by rotating the curve's image by an angle of $\pi$ about any of the three coordinate axes combined with an appropriate (dihedral) transformation of the periodic domain $\mathbb{R} / \mathbb{Z}$ of the curve. All this is made precise in Sect. 3; see the examples in Figs. 3 and 4 for a preliminary impression of $D_{2}$-symmetric curves. In particular, Fig. 4 depicts a dihedrally symmetric torus knot of class $\mathcal{T}(2,5)$ constructed in Example 3.10 with a method which works for any odd $b \in \mathbb{Z} \backslash\{1,-1\}$, so that the torus knot classes $\mathcal{T}(2, b)$ satisfy the hypothesis of Theorem 1.1 .

\footnotetext{
3 There are different definitions of a symmetry group for knots in the literature, cf. [1,14,28] and references therein. In the present paper we consider Euclidean symmetries of an actual space curve.
} 
Corollary 1.2 (Existence of symmetric critical torus knots) Let $b \in \mathbb{Z} \backslash\{-1,1\}$ be odd. Then for every $\vartheta>0$ there exists an arclength parametrized torus knot $\Gamma_{\vartheta} \in$ $\mathscr{C}(\mathcal{T}(2, b))$ with dihedral symmetry, which is critical for the constrained minimization $\operatorname{problem}\left(\mathrm{P}_{\vartheta}\right)$ for $\mathcal{K}=\mathcal{T}(2, b)$.

Apart from the $D_{2}$-symmetry these existence results contain no information about the actual shape of the critical knots $\Gamma_{\vartheta}$. In fact, there is a large variety of possible shapes of dihedrally symmetric curves. In Lemma 3.5 we provide a general mechanism how to construct space curves with dihedral symmetry from just one arc satisfying rather mild conditions on its endpoints. To obtain more specific information on the shape of the symmetric critical points $\Gamma_{\vartheta}$ obtained in Theorem 1.1 and Corollary 1.2 it seems hard to exploit the variational equation (1.5) because of the complicated differential $D \mathrm{TP}_{q}$ of the non-local tangent-point energy $\mathrm{TP}_{q}$ as part of the total energy $E_{\vartheta}$. Following the idea in [24] we study the limit $\vartheta \rightarrow 0$ instead, to obtain limit configurations whose shape can then be analyzed more easily to yield the approximative shape of the $D_{2}$-symmetric critical knots $\Gamma_{\vartheta}$ for small $\vartheta>0$.

Theorem 1.3 (Existence of symmetric elastic knots) Let $\mathcal{K}$ be a fixed knot class that contains a $D_{2}$-symmetric representative in $\mathscr{C}(\mathcal{K})$, and consider a sequence $\vartheta_{j} \rightarrow 0$ and the corresponding $\left(\mathrm{P}_{\vartheta_{j}}\right)$-critical $D_{2}$-symmetric knots $\Gamma_{\vartheta_{j}}$ obtained in Theorem 1.1. Then there exists an arclength parametrized curve $\Gamma_{0} \in W^{2,2}\left(\mathbb{R} / \mathbb{Z}, \mathbb{R}^{3}\right)$ with dihedral symmetry, and a subsequence $\left(\Gamma_{\vartheta_{j_{k}}}\right)_{k} \subset\left(\Gamma_{\vartheta_{j}}\right)_{j}$ such that the $\Gamma_{\vartheta_{j_{k}}}$ converge weakly in $W^{2,2}$ and strongly in $C^{1}$ to $\Gamma_{0}$ as $k \rightarrow \infty$. Moreover,

$$
E\left(\Gamma_{0}\right) \leq E(\beta) \text { for all } D_{2} \text {-symmetric } \beta \in \mathscr{C}(\mathcal{K})
$$

Definition 1.4 (Symmetric elastic knots) Any such curve $\Gamma_{0}$ obtained in Theorem 1.3 is called a dihedral (or $D_{2-}$ ) elastic knot for $\mathcal{K}$.

\section{Shapes of symmetric elastic knots}

The unknot class and the torus knot class $\mathcal{T}(2, b)$ for any odd $b \in \mathbb{Z} \backslash\{1,-1\}$ satisfy the hypothesis of Theorem 1.3; see Examples 3.8 and 3.10. Consequently, there are $D_{2}$-elastic knots for the unknot class and for $\mathcal{T}(2, b)$, and we can determine their shapes, which also turn out to characterize these knot classes.

Theorem 1.5 (The $D_{2}$-elastic unknot) Up to reparametrization and isometry, the only $D_{2}$-elastic unknot is the once covered circle of length one. Moreover, if a $D_{2}$-elastic knot for some knot class $\mathcal{K}$ is the once covered circle, then $\mathcal{K}$ is the unknot class. Only the unknot class $\mathcal{K}$ satisfies

$$
\inf _{\substack{\beta \in \mathscr{C}(\mathcal{K}) \\ \beta \text { is } D_{2} \text {-symmetric }}} E(\beta)=\inf _{\substack{\beta \in W^{2,2} \\ \mathscr{L}(\beta)=1}} E(\beta)=(2 \pi)^{2} .
$$

If we have specific information about the infimal bending energy on non-trivial knots with dihedral symmetry, then we can identify the shape of the corresponding 
$D_{2}$-elastic knot. To make this more precise, recall that the natural lower bound for the total curvature $\mathrm{TC}(\gamma):=\int_{\gamma} \kappa d s$ is $2 \pi$ by virtue of Fenchel's theorem [22]. Applying Hölder's inequality it transfers to the natural lower bound $(2 \pi)^{2}$ for the bending energy of closed curves of length one. Therefore, (1.7) is in fact equivalent to $\inf \left\{E(\beta) \mid \beta \in \mathscr{C}(\mathcal{K}), \beta\right.$ is $D_{2}$-symmetric $\} \leq(2 \pi)^{2}$. In case of non-trivial knots, the total curvature is bounded below by $4 \pi$ according to the famous result of Fáry and Milnor (see $[21,30]$ ) which gives rise to the lower bound $(4 \pi)^{2}$ for the bending energy for any non-trivial knot class $\mathcal{K}$.

Knot classes $\mathcal{K}$ for which the infimal bending energy equals the natural lower bound $(4 \pi)^{2}$, i.e., for which

$$
\inf _{\substack{\beta \in \mathscr{C}(\mathcal{K}) \\ \beta \text { is } D_{2} \text {-symmetric }}} E(\beta)=(4 \pi)^{2}=\inf _{\mathscr{C}(\mathcal{K})} E(\cdot)
$$

are of particular interest, since they provide the variational problem with a high degree of rigidity; see Theorem 4.5.

In Sect. 5 we analyze a sequence of specific $D_{2}$-symmetric $(2, b)$-torus knots like in Fig. 4 to show that all torus knot classes $\mathcal{T}(2, b)$ for odd integers $b \in \backslash\{-1,1\}$ satisfy condition (1.8). It actually turns out that there are no other knot classes satisfying (1.8). This leads to the following central characterization of $D_{2}$-elastic $(2, b)$-torus knots.

Theorem 1.6 ( $D_{2}$-elastic $(2, b)$-torus knots) The following statements hold up to isometry and reparametrization.

(i) The unique $D_{2}$-elastic $(2, b)$-torus knot for any odd $b \in \mathbb{Z} \backslash\{1,-1\}$ is the tangential pair of co-planar circles with exactly one point in common, denoted by $\mathrm{tpc}_{\pi}$. Any sequence of $D_{2}$-symmetric $E_{\vartheta}$-critical $(2, b)$-torus knots $\Gamma_{\vartheta}$ converges strongly in $W^{2,2}$ to $\operatorname{tpc}_{\pi}$ as $\vartheta \rightarrow 0$.

(ii) If a $D_{2}$-elastic knot for some knot class $\mathcal{K}$ is $\operatorname{tpc}_{\pi}$ then $\mathcal{K}=\mathcal{T}(2, b)$ for some odd $b \in \mathbb{Z} \backslash\{1,-1\}$.

(iii) If any non-trivial knot class $\mathcal{K}$ satisfies

$$
\inf _{\substack{\beta \in \mathscr{C}(\mathcal{K}) \\ D_{2} \text {-symmetric }}} E(\beta) \leq(4 \pi)^{2}
$$

then $\mathcal{K}=\mathcal{T}(2, b)$ for some odd $b \in \mathbb{Z} \backslash\{1,-1\}$.

Note that (1.8) and (1.8*) are in fact equivalent due to the Fáry-Milnor theorem.

The one-parameter family of tangential pairs of circles $\operatorname{tpc}_{\varphi}$ for $\varphi \in[0, \pi]$ was introduced in [24]; see Fig. 2. It consists of (isometric images of) pairs of circles each with radius $1 /(4 \pi)$ that intersect each other tangentially in at least one point. The parameter $\varphi$ describes the angle between the two planes spanned by the two circles. Only for $\varphi=0$ and $\varphi=\pi$ the two planes coincide, and the tangential pair of co-planar circles addressed in the rigidity result, Theorem 4.5, is (an isometric image of) $\operatorname{tpc}_{\pi}$; see Example 3.9. Part (i) of Theorem 1.6 improves the weak $W^{2,2}$-subconvergence of $D_{2}$ symmetric $E_{\vartheta}$-critical knots $\Gamma_{\vartheta}$, established in Theorem 1.3 for general knot classes $\mathcal{K}$, now to the strong convergence of every sequence of $D_{2}$-symmetric $E_{\vartheta}$-critical 
Fig. 2 The family of tangential pairs of circles tpc $\varphi$ parametrized by the opening angle $\varphi$ between the two planes containing the circles

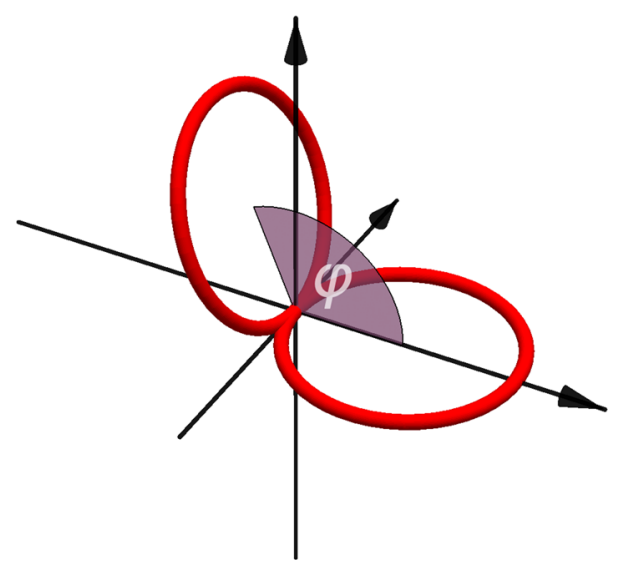

points $\Gamma_{\vartheta}$ to $\operatorname{tpc}_{\pi}$ for all torus knot classes $\mathcal{T}(2, b)$. Therefore, the limit curve tpc describes the approximate shape of the $\Gamma_{\vartheta}$ for small $\vartheta$, thus supporting the sometimes experimentally observed final configurations of the numerical gradient flow of Bartels et al. [7]; see Fig. 1 (right). However, as pointed out before, it seems unlikely that these $D_{2}$-symmetric critical points $\Gamma_{\vartheta}$ are local minimizers of $E_{\vartheta}$. To clarify this, one would need to analyze the second variation of the total energy containing the complicated non-local terms of the tangent-point energy $\mathrm{TP}_{q}$.

\section{Outline}

The paper is structured as follows. In Sect. 2.1 we briefly review the basics of the principle of symmetric criticality along the lines of the presentation in [25] and [26, Section 2], from which we adopted our approach to apply symmetric criticality to knotted space curves. The relevant facts about the tangent-point energy are presented in Sect. 2.2.

In Sect. 3 we discuss the group action in detail, show how to construct and characterize dihedrally symmetric space curves with increasing regularity (Definition 3.3 and Proposition 3.4). Of particular importance in our context are dihedrally symmetric circles and planar tangential pairs of circles whose exact location in space is determined in Corollary 3.11. We also prove in Lemma 3.12 that reparametrization to arclength does not destroy the symmetry and provide a sharp a priori estimate on the size of $D_{2}$ symmetric curves; see Lemma 3.13. We finally identify the suitable Banach manifold of $W^{2,2}$-regular knots in Lemma 3.14, on which the group $D_{2}$ acts in a sufficiently regular way required by Palais's symmetric criticality principle (Lemma 3.15).

Section 4 is devoted to proving the existence of the $D_{2}$-symmetric critical points as stated in Theorem 1.1, by first minimizing a rescaled total energy on symmetric knots (Theorem 4.1). By symmetric criticality these minimizers turn out to be critical points among all knots in the given knot class (Corollary 4.2) satisfying the desired Euler-Lagrange equation (1.5) as shown in Corollary 4.3. Moreover, the existence of $D_{2}$-symmetric elastic knots in any given tame knot class, i.e., the proof of Theorem 
1.3 , is established. The remainder of Sects. 4 and 5 deal with the shape of symmetric elastic knots, that is, the proofs of Theorems 1.5 and 1.6, the latter with the help of a general rigidity result (Theorem 4.5) for all knot classes satisfying (1.8) and an explicit convergence proof of the torus knots of Example 3.10 towards $\mathrm{tpc}_{\pi}$ carried out in Lemma 5.1.

In Sect. 6 we briefly touch on higher regularity of the symmetric critical knots obtained in Theorem 1.1, as well as on the question whether symmetric elastic knots are embedded. The concept of symmetric knots also applies to other symmetry classes than $D_{2}$. We give a brief outlook on the general case in that section.

The simulations shown in Figs. 1 and 5 have been carried out using the algorithm described in [6] which bases on an earlier work by Bartels [4]. We also refer to the app KNOTevolve [5].

\section{Preliminaries}

\subsection{Principle of symmetric criticality}

Let us briefly recall the notion of a group action on a Banach manifold modeled over a Banach space to describe symmetry in a mathematically rigorous way, cf. [33, pp. 19-20, 26].

Definition 2.1 For $k \in \mathbb{N}$ let $\mathscr{M}$ be a $C^{k}$-Banach manifold modeled over a Banach space $\mathscr{B}$, and suppose that $(G, \circ)$ is a group.

(i) If there is a mapping $\tau$ assigning to each $(g, x) \in G \times \mathscr{M}$ a point $\tau_{g}(x) \in \mathscr{M}$ such that

$$
\tau_{g \circ h}(x)=\tau_{g}\left(\tau_{h}(x)\right) \text { for all } g, h \in G, x \in \mathscr{M},
$$

then the group $G$ is said to act on $\mathscr{M}$, and $\tau$ is called a representation of $G$ on $\mathscr{M}$.

(ii) If for each $g \in G$ the mapping $\tau_{g}: \mathscr{M} \rightarrow \mathscr{M}$ is a $C^{k}$-diffeomorphism, then $\mathscr{M}$ is called a $G$-manifold (of class $C^{k}$ ). For an infinite Lie group $G$ one additionally requires that the representation $\tau$ is of class $C^{k}$ on $G \times \mathscr{M}$. If $\mathscr{M}$ is itself a Banach space and $\tau_{g}$ is linear then $\mathscr{M}$ is said to be a $G$-space.

(iii) For a $G$-manifold $\mathscr{M}$ the $G$-symmetric subset $\Sigma \subset \mathscr{M}$ is defined as

$$
\Sigma:=\left\{x \in \mathscr{M}: \tau_{g}(x)=x \text { for all } g \in G\right\}
$$

(iv) A function $F: \mathscr{M} \rightarrow \mathbb{R}$ is called $G$-invariant if and only if

$$
F\left(\tau_{g}(x)\right)=F(x) \quad \text { for all } g \in G, x \in \mathscr{M} \text {. }
$$

Palais proved the following symmetric criticality principle.

Theorem ([33, Theorem 5.4]) Suppose $G$ is a compact Lie group and $\mathscr{M}$ a $G$-manifold of class $C^{1}$ over the Banach space $\mathscr{B}$ with the non-empty $G$-symmetric subset $\Sigma \subset \mathscr{M}$, 
and let $F: \mathscr{M} \rightarrow \mathbb{R}$ be a $G$-invariant function of class $C^{1}$. Then $\Sigma$ is a $C^{1}$-submanifold of $\mathscr{M}$, and $x \in \Sigma$ is a critical point of $F$ if and only if $x$ is critical for the restricted functional $\left.F\right|_{\Sigma}: \Sigma \rightarrow \mathbb{R}$. That is, if $D\left(\left.F\right|_{\Sigma}\right)(x) v=0$ for all $v \in T_{x} \Sigma$, then also $D F(x) w=0$ for all $w \in T_{x} \mathscr{M}$.

As every finite group is a Lie group, cf., e.g., [16, p. 48, Example 5], we infer the following result that we apply in Sect. 4 to obtain dihedrally symmetric critical knots for the total energy $E_{\vartheta}$.

Corollary 2.2 (Symmetric criticality for finite groups) If $G$ is a finite group and $\mathscr{M}$ a $G$ manifold of class $C^{1}$ with non-empty $G$-symmetric subset $\Sigma \subset \mathscr{M}$, and if $F \in C^{1}(\mathscr{M})$ is $G$-invariant, then any critical point of $\left.F\right|_{\Sigma}$ is also a critical point of $F$.

Our choice of a Banach manifold will simply be an open subset $\Omega$ of a Banach space $\mathscr{B}$. This allows us to identify the differential of the energy $F: \Omega \rightarrow \mathbb{R}$ with the classic Fréchet-differential $D F(x): T_{x} \Omega \simeq \mathscr{B} \rightarrow T_{F(x)} \mathbb{R} \simeq \mathbb{R}$, which can be computed by means of the first variation

$$
D F(x) h=\delta F(x, h):=\lim _{\epsilon \rightarrow 0} \epsilon^{-1}[F(x+\epsilon h)-F(x)] \text { for } h \in \mathscr{B} .
$$

\subsection{Tangent-point energy}

As mentioned in the introduction, Gonzalez and Maddocks suggested in [27, p. 4773] to consider the tangent-point energy (1.4) as a candidate for a valuable knot energy. This was confirmed in the work of P. Strzelecki and the third author [38] starting at a rather low level of regularity with just rectifiable curves. In fact, arclength parametrizations $\Gamma \in C^{0,1}\left(\mathbb{R} / \mathbb{Z}, \mathbb{R}^{3}\right)$ of rectifiable curves with finite tangent-point energy $\operatorname{TP}_{q}(\Gamma)<\infty$ for $q \geq 2$ are either injective or they are multiple coverings of one-dimensional manifolds [38, Theorem 1.1]. In addition, such curves $\Gamma$ are of class $C^{1,1-(2 / q)}$ if $q>2$; see [38, Theorem 1.3].

In the present context, however, dealing with the bending energy $E$ we start at the already higher regularity level of closed $W^{2,2}$-curves which-according to the Morrey-Sobolev embedding theorem-are automatically of class $C^{1,1 / 2}$. Consequently, it suffices to review Blatt's regularity results $[9,10]$ on $C^{1}$-curves with finite tangent-point energy. ${ }^{4}$ One of the central results [9,10, Theorem 1.1] characterizes finite energy among embedded curves by fractional Sobolev regularity $W^{2-(1 / q), q}$. Here, we only need one part of that statement explicitly, in fact, in a slightly sharpened version for not necessarily arclength parametrized curves established in [26, Theorem 3.2 (ii)]. ${ }^{5}$

Theorem 2.3 Let $q \in(2, \infty)$ and suppose that $\gamma \in W^{2-(1 / q), q}\left(\mathbb{R} / \mathbb{Z}, \mathbb{R}^{3}\right)$ is injective and satisfies $\left|\gamma^{\prime}\right|>0$ on $\mathbb{R} / \mathbb{Z}$. Then $\mathrm{TP}_{q}(\gamma)<\infty$.

\footnotetext{
${ }^{4}$ Notice that the two-parameter family of energies $\mathrm{TP}^{(p, q)}$ considered in [10] contains the tangent-point energy, more precisely $\mathrm{TP}_{q}=2^{q} \mathrm{TP}^{(2 q, q)}$.

${ }^{5}$ For the proof of [26, Lemma A.1], which is used to establish [26, Theorem 3.2 (ii)], see the updated arXiv version.
} 
The other part of Blatt's characterization (or [38, Theorem 1.3] for that matter) can be used to quantify the degree of embeddedness for arclength parametrized curves $\Gamma: \mathbb{R} / \mathbb{Z} \rightarrow \mathbb{R}^{3}$ by means of the bi-Lipschitz constant

$$
\operatorname{BiLip}(\Gamma):=\inf _{\substack{s, t \in \mathbb{R} / \mathbb{Z} \\ s \neq t}} \frac{|\Gamma(s)-\Gamma(t)|}{|s-t|_{\mathbb{R} / \mathbb{Z}}}
$$

Lemma 2.4 (Bi-Lipschitz estimate for finite TP-energy [10, Proposition 2.7]) For any $q>2$ and $T>0$ there is a constant $C=C(q, T)>0$ such that any arclength parametrized and injective curve $\Gamma \in C^{0,1}\left(\mathbb{R} / \mathbb{Z}, \mathbb{R}^{3}\right)$ with $\mathrm{TP}_{q}(\Gamma) \leq T$ satisfies

$$
\operatorname{BiLip}(\Gamma) \geq C
$$

So far, we have reported on the effects that finite tangent-point energy has on the curve. Let us conclude this short review with continuity and regularity properties of the energy itself.

Theorem 2.5 (Regularity of the tangent-point energy) Let $q>2$. The tangent-point energy $\mathrm{TP}_{q}$ is sequentially lower semicontinuous with respect to $C^{1}$-convergence. Moreover, $\mathrm{TP}_{q}$ is continuously differentiable on regular embedded closed curves of fractional Sobolev regularity $W^{2-(1 / q), q}$.

Proof Lower semicontinuity of the tangent-point energy was shown in [37, p. 1513], whereas continuous differentiability was verified in [42] using the first variation formula in [10, Theorem 1.4] and the line of arguments used for the corresponding regularity statement for integral Menger curvature in [11, Theorem 3].

\subsection{Isotopy stability}

Several times in the proofs we will rely on the fact that knot classes are stable with respect to $C^{1}$-perturbations. Variants of the following statement can be found in [8, $18,19,34]$.

Lemma 2.6 (Ambient isotopy is open in $C^{1}$ ) For any embedded $\gamma \in C^{1}\left(\mathbb{R} / \ell \mathbb{Z}, \mathbb{R}^{3}\right.$ ) there is an $\varepsilon>0$ such that any $\tilde{\gamma} \in C^{1}\left(\mathbb{R} / \ell \mathbb{Z}, \mathbb{R}^{3}\right)$ with $\|\tilde{\gamma}-\gamma\|_{C^{1}}<\varepsilon$ is also embedded and belongs to the same knot class as $\gamma$.

\section{Group action on parametrized curves}

In order to describe the dihedral symmetry of parametrized closed curves $\gamma$ : $\mathbb{R} / \ell \mathbb{Z} \rightarrow \mathbb{R}^{3}$ we use two different representations of the dihedral group $D_{2}:=\left\{d_{0} \equiv\right.$ $\left.e, d_{1}, d_{2}, d_{3}\right\}$ with the multiplication table depicted in Table 1 , where $e$ denotes the identity element. Namely, in view of the symmetry of the curves' images we consider the subgroup $\left\{\mathrm{Id}_{\mathbb{R}^{3}} \equiv R_{0}, R_{1}, R_{2}, R_{3}\right\} \subset S O(3)$ containing the rotations $R_{i}$ about 
Table 1 The multiplication table of the dihedral group $D_{2}$

\begin{tabular}{lllll}
\hline & $e$ & $d_{1}$ & $d_{2}$ & $d_{3}$ \\
\hline$e$ & $e$ & $d_{1}$ & $d_{2}$ & $d_{3}$ \\
$d_{1}$ & $d_{1}$ & $e$ & $d_{3}$ & $d_{2}$ \\
$d_{2}$ & $d_{2}$ & $d_{3}$ & $e$ & $d_{1}$ \\
$d_{3}$ & $d_{3}$ & $d_{2}$ & $d_{1}$ & $e$ \\
\hline
\end{tabular}

the coordinate axes $\mathbb{R} \mathbf{e}_{i}$ for $i=1,2,3$, with rotational angle $\pi$, that is, written as matrices with respect to the standard coordinate basis $\left\{\mathbf{e}_{\mathbf{1}}, \mathbf{e}_{\mathbf{2}}, \mathbf{e}_{\mathbf{3}}\right\} \subset \mathbb{R}^{3}$,

$$
R_{1}:=\left(\begin{array}{ccc}
1 & 0 & 0 \\
0 & -1 & 0 \\
0 & 0 & -1
\end{array}\right), R_{2}:=\left(\begin{array}{ccc}
-1 & 0 & 0 \\
0 & 1 & 0 \\
0 & 0 & -1
\end{array}\right), R_{3}:=\left(\begin{array}{ccc}
-1 & 0 & 0 \\
0 & -1 & 0 \\
0 & 0 & 1
\end{array}\right)
$$

To take into account the curves' parametrizations, we use in addition the mappings $\psi_{i}^{\ell}: \mathbb{R} / \ell \mathbb{Z} \rightarrow \mathbb{R} / \ell \mathbb{Z}$ on the periodic domain $\mathbb{R} / \ell \mathbb{Z}$, defined as

$$
\psi_{i}^{\ell}(t):= \begin{cases}t \quad(\bmod \ell) & \text { for } i=0 \\ -t+\frac{\ell}{2}(\bmod \ell) & \text { for } i=1 \\ t-\frac{\ell}{2}(\bmod \ell) & \text { for } i=2 \\ -t+\ell \quad(\bmod \ell) & \text { for } i=3\end{cases}
$$

It is easy to check that for all mutually distinct $i, j, k \in\{1,2,3\}$ the following identities hold.

$$
\begin{array}{rll}
R_{i} \circ R_{i}=\operatorname{Id}_{\mathbb{R}^{3}} & \text { and } \quad \psi_{i}^{\ell} \circ \psi_{i}^{\ell}=\operatorname{Id}_{\mathbb{R} / \ell \mathbb{Z}}, \\
R_{i} \circ R_{j}=R_{k} & \text { and } \quad \psi_{i}^{\ell} \circ \psi_{j}^{\ell}=\psi_{k}^{\ell}, \\
\left.R_{i}\right|_{\mathbb{R} \mathbf{e}_{i}}=\operatorname{Id}_{\mathbb{R} \mathbf{e}_{i}} & \text { and } & \left.R_{i}\right|_{\mathbb{R} \mathbf{e}_{k}}=\left.R_{j}\right|_{\mathbb{R} \mathbf{e}_{k}}=-\left.\operatorname{Id}\right|_{\mathbb{R} \mathbf{e}_{k}} .
\end{array}
$$

Now we define how $D_{2}$ acts on the Banach space $C^{0}\left(\mathbb{R} / \mathbb{Z}, \mathbb{R}^{3}\right)$ of continuously parametrized closed curves (equipped with the norm $\|\cdot\|_{C^{0}}$ ).

Definition 3.1 Let $\tau^{\ell}: D_{2} \times C^{0}\left(\mathbb{R} / \ell \mathbb{Z}, \mathbb{R}^{3}\right) \rightarrow C^{0}\left(\mathbb{R} / \ell \mathbb{Z}, \mathbb{R}^{3}\right)$, mapping $\left(d_{i}, \gamma\right) \mapsto$ $\tau_{d_{i}}^{\ell}(\gamma)$ for $d_{i} \in D_{2}, i=0,1,2,3$, and $\gamma \in C^{0}\left(\mathbb{R} / \ell \mathbb{Z}, \mathbb{R}^{3}\right)$, be given by

$$
\tau_{d_{i}}^{\ell}(\gamma)(t):=R_{i} \circ \gamma\left(\psi_{i}^{\ell}(t)\right) \text { for } t \in \mathbb{R} / \ell \mathbb{Z}, i=0,1,2,3
$$

Lemma $3.2\left(C^{0}\left(\mathbb{R} / \ell \mathbb{Z}, \mathbb{R}^{3}\right)\right.$ is a smooth $D_{2}$-space) The mapping $\tau^{\ell}$ acts on $C^{0}\left(\mathbb{R} / \ell \mathbb{Z}, \mathbb{R}^{3}\right)$, and under this action $C^{0}\left(\mathbb{R} / \ell \mathbb{Z}, \mathbb{R}^{3}\right)$ becomes a smooth $D_{2}$-space.

Proof It is obvious that $\tau_{d_{i}}^{\ell}(\gamma) \in C^{0}\left(\mathbb{R} / \ell \mathbb{Z}, \mathbb{R}^{3}\right)$ for each $i=0,1,2,3$, and $\gamma \in$ $C^{0}\left(\mathbb{R} / \ell \mathbb{Z}, \mathbb{R}^{3}\right)$, since any rotation in the image and affine linear transformation of the periodic domain neither changes the $C^{0}$-regularity nor the $\ell$-periodicity. 
According to the multiplication table of the group $D_{2}$ (see Table 1) and by the properties (3.3)-(3.5) we have

$$
\tau_{d_{i} \circ d_{i}}^{\ell}(\gamma)(t) \stackrel{\text { Table } 1}{=} \tau_{e}^{\ell}(\gamma)(t) \stackrel{(3.6),(3.2)}{=} \gamma(t) \text { for all } t \in \mathbb{R} / \ell \mathbb{Z}, i=0,1,2,3 .
$$

On the other hand, again by (3.6) and (3.3),

$$
\begin{aligned}
\tau_{d_{i}}^{\ell}\left(\tau_{d_{i}}^{\ell}(\gamma)\right)(t) & \stackrel{(3.6)}{=} R_{i} \circ\left(\tau_{d_{i}}^{\ell}(\gamma)(\cdot)\right)\left(\psi_{i}^{\ell}(t)\right) \\
& \stackrel{(3.6)}{=} R_{i} \circ R_{i} \circ \gamma\left(\psi_{i}^{\ell}\left(\psi_{i}^{\ell}(t)\right)\right) \stackrel{(3.3)}{=} \gamma(t) \text { for all } t \in \mathbb{R} / \ell \mathbb{Z}, i=0,1,2,3,
\end{aligned}
$$

which proves the homomorphism property (2.1) for identical group elements in $D_{2}$. For $i \neq j=0$ there is nothing to prove since $j=0$ corresponds to the identity elements in the respective representations of $D_{2}$. For $i \neq j, i, j \in\{1,2,3\}$ we use the multiplication rules in Table 1 and the definition (3.6) to find, on the one hand,

$$
\tau_{d_{i} \circ d_{j}}^{\ell}(\gamma)(t) \stackrel{\text { Table } 1}{=} \tau_{d_{k}}^{\ell}(\gamma) \stackrel{(3.6)}{=} R_{k} \circ \gamma\left(\psi_{k}^{\ell}(t)\right),
$$

whereas (3.6), as well as (3.4) lead to

$$
\begin{aligned}
\tau_{d_{i}}^{\ell}\left(\tau_{d_{j}}^{\ell}(\gamma)\right)(t) & \stackrel{(3.6)}{=} \tau_{d_{i}}^{\ell}\left(R_{j} \circ \gamma\left(\psi_{j}^{\ell}(\cdot)\right)\right)(t) \\
& \stackrel{(3.6)}{=} R_{i} \circ R_{j} \circ \gamma\left(\psi_{j}^{\ell}\left(\psi_{i}^{\ell}(t)\right)\right) \stackrel{(3.4)}{=} R_{k} \circ \gamma\left(\psi_{k}^{\ell}(t)\right),
\end{aligned}
$$

which equals the expression in (3.7). We have shown so far that $\tau$ is indeed a representation of $D_{2}$ on $C^{0}\left(\mathbb{R} / \mathbb{Z}, \mathbb{R}^{3}\right)$. Since $\tau_{d_{i}}^{\ell}(\sigma \gamma+\eta)(t)=R_{i} \circ(\sigma \gamma+\eta)\left(\psi_{i}^{\ell}(t)\right)=$ $\sigma R_{i} \circ \gamma\left(\psi_{i}^{\ell}(t)\right)+R_{i} \circ \eta\left(\psi_{i}^{\ell}(t)\right)=\sigma \tau_{d_{i}}^{\ell}(\gamma)+\tau_{d_{i}}^{\ell}(\eta)$ for all $\gamma, \eta \in C^{0}\left(\mathbb{R} / \ell \mathbb{Z}_{\mathbb{R}^{3}}\right)$ and $\sigma \in \mathbb{R}$, one finds that $\tau_{d_{i}}^{\ell}: C^{0}\left(\mathbb{R} / \ell \mathbb{Z}, \mathbb{R}^{3}\right) \rightarrow C^{0}\left(\mathbb{R} / \ell \mathbb{Z}, \mathbb{R}^{3}\right)$ is linear for all $i=0,1,2,3$, so that the Banach space $C^{0}\left(\mathbb{R} / \ell \mathbb{Z}, \mathbb{R}^{3}\right)$ is indeed a $D_{2}$-space in the sense of Definition 2.1, part (ii).

Symmetric curves are of particular interest here, which are defined as follows.

Definition 3.3 (Dihedrally symmetric curves) A curve is called dihedrally symmetric or $D_{2}$-symmetric if it belongs to the $D_{2}$-symmetric set

$$
\Sigma^{\ell}:=\left\{\gamma \in C^{0}\left(\mathbb{R} / \ell \mathbb{Z}, \mathbb{R}^{3}\right): 0<\mathscr{L}(\gamma)<\infty, \tau_{d_{i}}^{\ell}(\gamma)=\gamma \text { for all } d_{i} \in D_{2}\right\}
$$

Now we provide a method to systematically construct examples of $D_{2}$-symmetric curves (of finite length). For that we glue copies of an open or closed $\operatorname{arc} \alpha:[0, \ell / 4] \rightarrow$ $\mathbb{R}^{3}$ of finite length together to obtain a mapping $g:[0, \ell) \rightarrow \mathbb{R}^{3}$ as

$$
g(t):= \begin{cases}\alpha(t) & \text { for } t \in[0, \ell / 4), \\ R_{1} \circ \alpha\left(\psi_{1}^{\ell}(t)\right) & \text { for } t \in[\ell / 4, \ell / 2), \\ R_{2} \circ \alpha\left(\psi_{2}^{\ell}(t)\right) & \text { for } t \in[\ell / 2,3 \ell / 4), \\ R_{3} \circ \alpha\left(\psi_{3}^{\ell}(t)\right) & \text { for } t \in[3 \ell / 4, \ell),\end{cases}
$$


and investigate first under which circumstances this glueing process leads to a closed curve with a certain regularity. Notice that $\psi_{i}^{\ell}([i \ell / 4,(i+1) \ell / 4))=(0, \ell / 4]$ for $i=1,3$, and $\psi_{2}^{\ell}([\ell / 2,3 \ell / 4))=[0, \ell / 4)$ by definition of the $\psi_{j}^{\ell}$ in $(3.2)$, so that $g$ in (3.9) is well-defined. It turns out that this construction does not only produce examples of $D_{2}$-symmetric curves but also characterizes this symmetry ${ }^{6}$.

Proposition 3.4 (i) $\gamma \in \Sigma^{\ell}$ if and only if there exists an $\operatorname{arc} \alpha \in C^{0}\left([0, \ell / 4], \mathbb{R}^{3}\right)$ of positive and finite length satisfying

$$
\alpha(0) \in \mathbb{R} \mathbf{e}_{3} \quad \text { and } \quad \alpha(\ell / 4) \in \mathbb{R} \mathbf{e}_{1},
$$

such that $\gamma$ coincides with the $\ell$-periodic extension of $g$ defined in (3.9). In particular, the points $\gamma(0)$ and $\gamma(\ell / 2)$ are contained in $\mathbb{R} \mathbf{e}_{3}$, whereas $\gamma(\ell / 4)$ and $\gamma(3 \ell / 4)$ are contained in $\mathbb{R} \mathbf{e}_{\mathbf{1}}$.

(ii) $\gamma \in \Sigma^{\ell} \cap W^{1, p}\left(\mathbb{R} / \ell \mathbb{Z}, \mathbb{R}^{3}\right)$ for some $p \in[1, \infty]$ if and only if $\gamma=g$, where $\alpha$ is of class $W^{1, p}$ and satisfies (3.10).

(iii) $\gamma \in \Sigma^{\ell} \cap C^{1}\left(\mathbb{R} / \ell \mathbb{Z}, \mathbb{R}^{3}\right)$ if and only if $\gamma=g$ in the sense of (i), where $\alpha \in$ $C^{1}\left([0, \ell / 4], \mathbb{R}^{3}\right)$ satisfies $(3.10)$ and

$$
\alpha^{\prime}(0) \in \operatorname{span}\left\{\mathbf{e}_{1}, \mathbf{e}_{2}\right\} \quad \text { and } \quad \alpha^{\prime}(\ell / 4) \in \operatorname{span}\left\{\mathbf{e}_{2}, \mathbf{e}_{3}\right\} .
$$

Moreover, $\gamma \in \Sigma^{\ell} \cap W^{2, p}\left(\mathbb{R} / \ell \mathbb{Z}, \mathbb{R}^{3}\right)$ for some $p \in[1, \infty]$ if and only if, in addition to the above properties, $\alpha$ is of class $W^{2, p}$.

(iv) Let $S_{i}$ be the reflection in the coordinate plane $\mathbf{e}_{i}^{\perp}$ for $i=1,2,3$. Then, $S_{i}\left(\Sigma^{\ell}\right) \subset$ $\Sigma^{\ell}, S_{i}\left(\Sigma^{\ell} \cap C^{1}\right) \subset \Sigma^{\ell} \cap C^{1}$, and $S_{i}\left(\Sigma^{\ell} \cap W^{k, p}\right) \subset \Sigma^{\ell} \cap W^{k, p}$ for $p \in[1, \infty]$, $k=1,2$.

The proof of this proposition will follow from the following partial results.

Lemma 3.5 (Glueing produces closed curves) Suppose $\alpha \in C^{0}\left([0, \ell / 4], \mathbb{R}^{3}\right)$ has length $\mathscr{L}(\alpha) \in(0, \infty)$, then the mapping $g$ defined according to (3.9) has length $\mathscr{L}(g)=4 \mathscr{L}(\alpha)$. Moreover, $g$ is closed and continuous, i.e., of class $C^{0}\left(\mathbb{R} / \ell \mathbb{Z}, \mathbb{R}^{3}\right)$ if and only if (3.10). Finally, if $\alpha$ is continuously differentiable, the curve $g$ is of class $C^{1}\left(\mathbb{R} / \ell \mathbb{Z}, \mathbb{R}^{3}\right)$ if and only if in addition to (3.10) the tangents of $\alpha$ satisfy (3.11).

Proof Since $\alpha$ is continuous one has $\mathscr{L}_{[0, \ell / 4)}(g)=\mathscr{L}(\alpha)$ (see, e.g., [31, VIII, Section 5, Theorem 1, p. 223]), and because the rotated images of $\alpha$ have the same length as $\alpha$, the statement about $\mathscr{L}(g)$ is immediate.

The $\ell$-periodic extension of the piecewise defined curve $g$ is continuous if and only if the following four identities hold true:

$$
\begin{gathered}
g(0)=\lim _{t \nearrow \ell} R_{3} \circ \alpha(-t+\ell), \quad g(\ell / 4)=\lim _{t \nearrow \ell / 4} \alpha(t), \\
g(\ell / 2)=\lim _{t \nearrow \ell / 2} R_{1} \circ \alpha(-t+\ell / 2), \quad g(3 \ell / 4)=\lim _{t \nearrow 3 \ell / 4} R_{2} \circ \alpha(t-\ell / 2),
\end{gathered}
$$

\footnotetext{
6 Fixing the rotational axes with (3.1) and the corresponding parameter transformations in (3.2) enforces a rigidity on the class of $D_{2}$-symmetric curves, which is reflected in the statements of Proposition 3.4 and Corollary 3.11 below.
} 
where we have already plugged in the definition (3.9) in the respective limits on the right-hand sides. Using the continuity of $\alpha$ on the right-hand side we obtain the conditions $\alpha(0)=R_{3} \circ \alpha(0)$ and $\alpha(\ell / 4)=R_{1} \circ \alpha(\ell / 4)$ that are equivalent to (3.12), and with the help of (3.3) and (3.4) exactly the same conditions equivalent to (3.13). From (3.5) we infer $\operatorname{ker}\left(\mathrm{Id}-R_{i}\right)=\mathbb{R} \mathbf{e}_{i}$ for $i=1,2,3$, which implies that these identities on the endpoints $\alpha(0)$ and $\alpha(\ell / 4)$ are equivalent to (3.10).

Provided that $\alpha$ is continuosly differentiable, $C^{1}$-regularity of $g$ is equivalent to the pointwise conditions (3.12) and (3.13) in combination with the tangential conditions

$$
\begin{array}{cl}
g^{\prime}(0)=\lim _{t \nearrow \ell} R_{3} \circ\left(-\alpha^{\prime}(-t+\ell)\right), & g^{\prime}(\ell / 4)=\lim _{t \nearrow \ell / 4} \alpha^{\prime}(t), \\
g^{\prime}(\ell / 2)=\lim _{t \nearrow \ell / 2} R_{1} \circ\left(-\alpha^{\prime}(-t+\ell / 2)\right), & g^{\prime}(3 \ell / 4)=\lim _{t \nearrow 3 \ell / 4} R_{2} \circ \alpha^{\prime}(t-\ell / 2),
\end{array}
$$

where the minus signs in the respective left equations are a consequence of the chain rule. Now by continuity of $\alpha^{\prime}$ we obtain from (3.14) the equivalent conditions $\alpha^{\prime}(0)=$ $-R_{3} \circ \alpha^{\prime}(0)$ and $\alpha^{\prime}(\ell / 4)=-R_{1} \circ \alpha^{\prime}(\ell / 4)$. From $\operatorname{ker}\left(\operatorname{Id}+R_{i}\right)=\operatorname{span}\left\{\mathbf{e}_{j}, \mathbf{e}_{k}\right\}$ we deduce that they are equivalent to (3.11). Exploiting (3.15) again with the help of (3.3) and (3.4) leads to the same conditions on $\alpha^{\prime}(0)$ and $\alpha^{\prime}(\ell / 4)$.

From the Morrey-Sobolev embedding in one dimension together with well-known glueing properties for Sobolev functions [2, E3.6 \& E3.7] one readily obtains the following corollary.

Corollary 3.6 [Glueing Sobolev arcs] If $\alpha \in W^{1, p}\left((0, \ell / 4), \mathbb{R}^{3}\right)$ for any $p \in[1, \infty]$, then $g$ defined in (3.9) is a closed curve of class $W^{1, p}\left(\mathbb{R} / \ell \mathbb{Z}, \mathbb{R}^{3}\right)$ if and only if the continuous representative of $\alpha$ satisfies (3.10). If $\alpha \in W^{2, p}\left((0, \ell / 4), \mathbb{R}^{3}\right)$, then $g$ is a closed curve of class $W^{2, p}\left(\mathbb{R} / \ell \mathbb{Z}, \mathbb{R}^{3}\right)$ if and only if the $C^{1}$-representative of $\alpha$ satisfies (3.10) and (3.11).

Now we are in the position to prove that the constructed curve $g$ in (3.9) is also $D_{2}$-symmetric.

Lemma 3.7 ( $D_{2}$-symmetric curves) If $\alpha \in C^{0}\left([0, \ell / 4], \mathbb{R}^{3}\right)$ has finite and positive length and satisfies (3.10), then the curve $g$ defined in (3.9) is $D_{2}$-symmetric, that is, $g \in \Sigma^{\ell}$. If $\alpha$ is of class $W^{1, p}$ for some $p \in[1, \infty]$, then so is the $D_{2}$-symmetric curve $g$, and if $\alpha \in C^{1}\left([0, \ell / 4], \mathbb{R}^{3}\right)$ or if $\alpha$ is of class $W^{2, p}$, and satisfies (3.11) in addition, then $g$ is a $D_{2}$-symmetric closed curve of class $C^{1}$, or $W^{2, p}$, respectively.

Proof The regularity statements follow from Lemma 3.5 and Corollary 3.6, so it suffices to prove $D_{2}$-symmetry, for which we merely need to show that

$$
\tau_{d_{i}}^{\ell}(g)=g \quad \text { for } i=1,2,3 .
$$

We only treat the case $i=1$ in full detail, the cases $i=2,3$ are very similar.

For $t \in[0, \ell / 4)$ we have $\psi_{1}^{\ell}(t) \in(\ell / 4, \ell / 2]$, and therefore by definition of $g$

$$
\tau_{d_{1}}^{\ell}(g)(t) \stackrel{(3.6)}{=} R_{1} \circ g\left(\psi_{1}^{\ell}(t)\right) \stackrel{(3.9)}{=} R_{1} \circ R_{1} \circ \alpha\left(\psi_{1}^{\ell} \circ \psi_{1}^{\ell}(t)\right) \stackrel{(3.3)}{=} \alpha(t)=g(t)
$$


Notice that we have also used the continuity of $g$ established in Lemma 3.5 to treat the parameter $t=0$, since $\psi_{1}^{\ell}(0)=\ell / 2$, so that

$$
g\left(\psi_{1}^{\ell}(0)\right)=g(\ell / 2)=\lim _{t \nearrow \ell / 2} g(t) \stackrel{(3.9)}{=} \lim _{t \nearrow \ell / 2} R_{1} \circ \alpha\left(\psi_{1}^{\ell}(t)\right)=R_{1} \circ \alpha(0) .
$$

For $t \in[\ell / 4, \ell / 2)$ one has $\psi_{1}^{\ell}(t) \in(0, \ell / 4]$, so that

$$
\tau_{d_{1}}^{\ell}(g)(t) \stackrel{(3.6)}{=} R_{1} \circ g\left(\psi_{1}^{\ell}(t)\right) \stackrel{(3.9)}{=} R_{1} \circ \alpha\left(\psi_{1}^{\ell}(t)\right) \stackrel{(3.9)}{=} g(t),
$$

where again, we have used the continuity of $g$ to treat $t=\ell / 4$ by means of $g\left(\psi_{1}^{\ell}(\ell / 4)\right)=g(\ell / 4)=\lim _{t} / \ell / 4 g(t)$ similarly as above.

For $t \in[\ell / 2,3 \ell / 4)$ we have $\psi_{1}^{\ell}(t) \in(-\ell / 4,0] \equiv(3 \ell / 4, \ell](\bmod \ell)$, so that

$$
\begin{aligned}
\tau_{d_{1}}^{\ell}(g)(t) & \stackrel{(3.6)}{=} R_{1} \circ g\left(\psi_{1}^{\ell}(t)\right) \stackrel{(3.9)}{=} R_{1} \circ R_{3} \circ \alpha\left(\psi_{3}^{\ell} \circ \psi_{1}^{\ell}(t)\right) \\
& \stackrel{(3.4)}{=} R_{2} \circ \alpha\left(\psi_{2}^{\ell}(t)\right) \stackrel{(3.9)}{=} g(t),
\end{aligned}
$$

where the parameter $t=\ell / 2$ was treated as before via $g\left(\psi_{1}^{\ell}(\ell / 2)\right)=g(0)=$ $\lim _{t \succ \ell} g(t)$. Finally, for $t \in[3 \ell / 4, \ell)$, one has $\psi_{1}^{\ell}(t) \in(-\ell / 2,-\ell / 4] \equiv(\ell / 2,3 \ell / 4]$ $(\bmod \ell)$, so that

$$
\begin{aligned}
\tau_{d_{1}}^{\ell}(g)(t) & \stackrel{(3.6)}{=} R_{1} \circ g\left(\psi_{1}^{\ell}(t)\right) \stackrel{(3.9)}{=} R_{1} \circ R_{2} \circ \alpha\left(\psi_{2}^{\ell} \circ \psi_{1}^{\ell}(t)\right) \\
& \stackrel{(3.4)}{=} R_{3} \circ \alpha\left(\psi_{3}^{\ell}(t)\right) \stackrel{(3.9)}{=} g(t),
\end{aligned}
$$

the parameter $t=3 \ell / 4$ treated by continuity of $g$ via $g\left(\psi_{1}^{\ell}(3 \ell / 4)\right)=g(-\ell / 4)=$ $g(3 \ell / 4)=\lim _{t / 3 \ell / 4} g(t)$ by $\ell$-periodicity of $g$.

Thus, we have finished the detailed argument for $i=1$. The case $i=3$ is analogous, and $i=2$ is somewhat simpler, since there is no inversion in the domain which saves us the additional continuity argument to treat the respective boundary parameters.

Now we can present the

Proof of Proposition 3.4 For part (i) assume that $\gamma \in \Sigma^{\ell}$ so $\gamma=R_{i} \circ \gamma\left(\psi_{i}^{\ell}(\cdot)\right)$ for $i=0,1,2,3$. Let $\alpha:=\left.\gamma\right|_{[0, \ell / 4]}$ and define $g$ according to (3.9). Then

$$
\begin{array}{r}
g(t)=R_{j} \circ \alpha\left(\psi_{j}^{\ell}(t)\right)=R_{j} \circ \gamma\left(\psi_{j}^{\ell}(t)\right)=\gamma(t) \\
\text { for } t \in[j \ell / 4,(j+1) \ell / 4), j=0,1,2,3 .
\end{array}
$$

The other implication in part (i) and also parts (ii) and (iii) follow from Lemmata 3.5 and 3.7, and Corollary 3.6. Finally, part (iv) follows from the characterizations of $\Sigma^{\ell}$, $\Sigma^{\ell} \cap C^{1}$ and $\Sigma^{\ell} \cap W^{k, p}$ for $k=1,2$ in terms of the generating arc $\alpha$, established in the previous parts (i)-(iii). These characterizations can be combined with the fact 


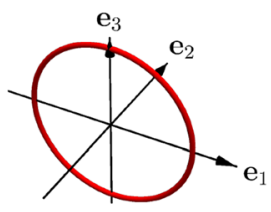

(A)

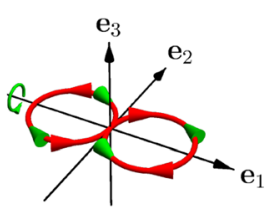

(B)

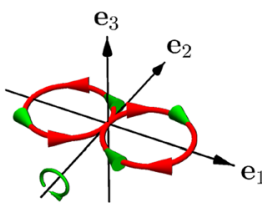

(C)

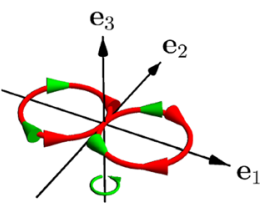

(D)

Fig. $3 D_{2}$-symmetric curves generated by (3.9). A The once covered circle in the $\mathbf{e}_{\mathbf{1}}$-e $\mathbf{3}$-plane from Example 3.8. B-D A tangential pair of co-planar circles exhibiting symmetries with respect to the coordinate axes as described in Example 3.9. $180^{\circ}$ rotations about the coordinate axes $\mathbb{R} \mathbf{e}_{\boldsymbol{i}}$ indicated in green are accompanied by parameter transformations $\psi_{i}^{\ell}$ that reverse orientation for $i=1,3$ in $\mathbf{B}$ and $\mathbf{D}$, while preserving it for $i=2$ in $\mathbf{C}$; see red and green arrows along the curves

that the conditions (3.10) and (3.11) on $\alpha$ are invariant under the reflections $S_{i}$ for $i=1,2,3$.

We use the glueing mechanism (3.9) to construct a few explicit examples of dihedrally symmetric curves parametrized on $\mathbb{R} / \ell \mathbb{Z}$.

Example 3.8 As a first generating arc $\alpha_{1} \in C^{\infty}\left([0, \ell / 4], \mathbb{R}^{3}\right)$ we choose a quadrant (i.e., one quarter of a circle) in the $\mathbf{e}_{1}$ - $\mathbf{e}_{3}$-plane with arclength $\ell / 4$, that is,

$$
\alpha_{1}(t):=\frac{\ell}{2 \pi}\left(\begin{array}{c}
\sin (2 \pi t / \ell) \\
0 \\
\cos (2 \pi t / \ell)
\end{array}\right) \quad \text { for } t \in[0, \ell / 4]
$$

so that $\alpha:=\alpha_{1}$ satisfies the conditions (3.10), (3.11), and the regularity assumptions of Lemma 3.5, Corollary 3.6, and Lemma 3.7. According to these results the curve $g \equiv g_{1}$ defined in (3.9) for this particular choice of $\alpha=\alpha_{1}$ is a $C^{1,1}$-closed and $D_{2}$-symmetric curve, that is, $g_{1} \in \Sigma^{\ell} \cap C^{1,1}\left(\mathbb{R} / \ell \mathbb{Z}, \mathbb{R}^{3}\right)$. It is easy to check that $g_{1}$ is the once covered circle whose parametrization equals (3.17) if one extends the domain of the latter to all of $[0, \ell]$, so $g_{1}$ is actually $C^{\infty}$ on $\mathbb{R} / \ell \mathbb{Z}$; see Fig. $3 \mathrm{~A}$.

Example 3.9 We construct a dihedrally symmetric tangential pair of co-planar circles with exactly one self-intersection point within the $\mathbf{e}_{\mathbf{1}}-\mathbf{e}_{\mathbf{2}}$-plane; see Fig. 3B-D. For that we take as a generating $\operatorname{arc} \alpha_{2} \in C^{\infty}\left([0, \ell / 4], \mathbb{R}^{3}\right)$ a semicircle of radius $\ell /(4 \pi)$. To be more precise, we set

$$
\alpha_{2}(t):=\frac{\ell}{4 \pi}\left(\begin{array}{c}
1-\cos (4 \pi t / \ell) \\
\sin (4 \pi t / \ell) \\
0
\end{array}\right) \quad \text { for } t \in[0, \ell / 4]
$$

and easily check that conditions (3.10) and (3.11) as well as the regularity assumptions of Lemma 3.5, Corollary 3.6, and Lemma 3.7 are satisfied for $\alpha:=\alpha_{2}$. Glueing according to (3.9) yields the $D_{2}$-symmetric tangential pair of co-planar circles with parametrization $g \equiv g_{2} \in \Sigma^{\ell} \cap C^{1,1}\left(\mathbb{R} / \ell \mathbb{Z}, \mathbb{R}^{3}\right)$, which in the case $\ell=1$ we also denote by $\operatorname{tpc}_{\pi}$. This curve has the same trace as the corresponding curve in [24, Formula (3.2) for $\varphi:=\pi$ ], only with reversed orientation. 

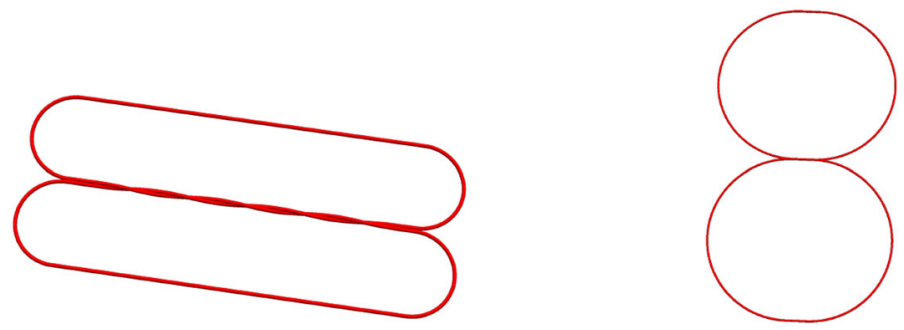

Fig. 4 Dihedrally symmetric torus knots of class $\mathcal{T}(2,5)$ constructed in Example 3.10 for $\epsilon=0.03$ (left) and for $\epsilon=0.003$ (right)

In a similar manner, we may construct a $\operatorname{tpc}_{\pi}$-curve in the $\mathbf{e}_{\mathbf{2}}$ - $\mathbf{e}_{\mathbf{3}}$-plane by letting

$$
\alpha_{2}(t):=\frac{\ell}{4 \pi}\left(\begin{array}{c}
0 \\
\sin (4 \pi t / \ell) \\
1+\cos (4 \pi t / \ell)
\end{array}\right) \quad \text { for } t \in[0, \ell / 4]
$$

We will show in Corollary 3.11 that (3.18) and (3.19) are the only two options for the generating arc to construct $D_{2}$-symmetric tangential pairs of circles tpc ${ }_{\pi}$.

Our third example produces dihedrally symmetric torus knots of class $\mathcal{T}(2, b)$ for any odd $b \in \mathbb{Z} \backslash\{1,-1\}$; see Fig. 4 .

Example 3.10 The generating curve $\alpha_{3}$ consists of a helical part $h$, which after the glueing forms together with its rotated copies a rational tangle that determines the knot class [1, Section 2.3], and a piece $\sigma$ of a stadium curve, which after the glueing closes the tangle to form the knot; see Fig. 4. For the precise formulas, which we are going to take up again in Sect. 5 to compute the infimal bending energy on $D_{2}$ symmetric torus knots, it suffices to consider a fixed odd integer $b \geq 3$. Indeed, the reflection of a $D_{2}$-symmetric representative of the torus knot class $\mathcal{T}(2, b)$ in a coordinate plane $\mathbf{e}_{\boldsymbol{i}}$ for $i=1,2,3$, produces a representative of $\mathcal{T}(2,-b)$ that still possesses the $D_{2}$-symmetry according to part (iv) of Proposition 3.4. We also fix two parameters $\epsilon, \varrho>0$, and define the helical part $h=h^{\epsilon}$ of the generating arc as

$$
h^{\epsilon}(t):=\left(\begin{array}{c}
\varrho(-1)^{(b-1) / 2} \sin \phi_{\epsilon}(t) \\
t \\
\varrho \cos \phi_{\epsilon}(t)
\end{array}\right) \quad \text { for } t \in[0, \infty)
$$

where $\phi_{\epsilon}(t):=\pi \cdot \phi(t / \epsilon)$ for the piecewise smooth parameter transformation $\phi \epsilon$ $C^{1,1}([0, \infty))$ given by

$$
\phi(t):= \begin{cases}t & \text { for } t \in\left[0, \frac{b-1}{2}\right], \\ \frac{b}{2}-\frac{1}{2}\left(t-\frac{b+1}{2}\right)^{2} & \text { for } t \in\left[\frac{b-1}{2}, \frac{b+1}{2}\right], \\ \frac{b}{2} & \text { for } t \geq \frac{b+1}{2}\end{cases}
$$


Note that

$$
h^{\varepsilon}\left(\frac{(b+1) \varepsilon}{2}\right)=\left(\begin{array}{c}
\varrho(-1)^{(b-1) / 2} \sin \frac{b \pi}{2} \\
\frac{(b+1) \varepsilon}{2} \\
0
\end{array}\right)=\left(\begin{array}{c}
\varrho \\
\frac{(b+1) \varepsilon}{2} \\
0
\end{array}\right) .
$$

The portion $\sigma=\sigma^{\epsilon}$ of a stadium curve of class $C^{1,1}$ consists of a semicircle of radius $r=r(\epsilon)$ satisfying

$$
(b+1) \epsilon+\pi r=\ell / 4,
$$

and a (short) straight segment attached in a $C^{1}$-manner to the semicircle. The precise definition is

$$
\sigma^{\epsilon}(t):= \begin{cases}\left(\begin{array}{c}
\varrho+r-r \cos \left(\frac{1}{r}(t-(b+1) \epsilon / 2)\right) \\
(b+1) \epsilon / 2+r \sin \left(\frac{1}{r}(t-(b+1) \epsilon / 2)\right) \\
0
\end{array}\right) & \text { for } t \in\left[\frac{(b+1) \epsilon}{2}, \frac{\ell}{4}-\frac{(b+1) \epsilon}{2}\right], \\
\left(\begin{array}{c}
\varrho+2 r \\
(\ell / 4)-t \\
0
\end{array}\right) & \text { for } t \in\left[\frac{\ell}{4}-\frac{(b+1) \epsilon}{2}, \frac{\ell}{4}\right] .\end{cases}
$$

The generating arc $\alpha_{3}^{\epsilon}$ for fixed $\epsilon>0$ satisfying (3.23) is now defined as

$$
\alpha_{3}^{\epsilon}(t):= \begin{cases}h^{\epsilon}(t) & \text { for } t \in\left[0, \frac{b+1}{2} \cdot \epsilon\right] \\ \sigma^{\epsilon}(t) & \text { for } t \in\left(\frac{b+1}{2} \cdot \epsilon, \frac{\ell}{4}\right]\end{cases}
$$

and one easily checks with the help of (3.23) and (3.22) that $\alpha_{3}^{\epsilon}$ itself is of class $C^{1,1}$ on $[0, \ell / 4]$. Moreover, $\alpha_{3}^{\epsilon}(0)=h^{\epsilon}(0)=(0,0, \varrho)^{T} \in \mathbb{R} \mathbf{e}_{3}$ and $\alpha_{3}^{\epsilon}(\ell / 4)=(\varrho+2 r, 0,0)^{T} \in \mathbb{R} \mathbf{e}_{1}$ as required in (3.10) of Lemma 3.5. Finally, $\left(\alpha_{3}^{\epsilon}\right)^{\prime}(0)=\left(\varrho(-1)^{(b-1) / 2} \pi / \epsilon, 1,0\right)^{T} \in \operatorname{span}\left\{\mathbf{e}_{1}, \mathbf{e}_{2}\right\}$ and $\left(\alpha_{3}^{\epsilon}\right)^{\prime}(\ell / 4)=(0,-1,0)^{T} \in$ $\operatorname{span}\left\{\mathbf{e}_{\mathbf{2}}, \mathbf{e}_{3}\right\}$, so that also (3.11) is satisfied. Therefore, all assumptions of Lemma 3.5, Corollary 3.6, and Lemma 3.7 are satisfied, so that the glueing (3.9) yields a $D_{2-}$ symmetric torus knot $g_{3}^{\epsilon} \in \Sigma^{\ell} \cap C^{1,1}\left(\mathbb{R} / \ell \mathbb{Z}, \mathbb{R}^{3}\right)$ representing the knot class $\mathcal{T}(2, b)$.

We establish in Section 5 the strong $W^{2,2}$-convergence of these torus knots $g_{3}^{\epsilon}$ for $\ell=1$ to the tangential pair of co-planar circles constructed in Example 3.9, i.e., to $\operatorname{tpc}_{\pi}$ as $\epsilon \rightarrow 0$.

In light of the above examples, it is in order to ask about the location of the dihedrally symmetric curves appearing in the main theorems, namely the round circle and the tangential pair of circles $\operatorname{tpc}_{\pi}$.

Corollary $3.11\left(D_{2}\right.$-symmetric circle and $\left.\mathrm{tpc}_{\pi}\right)$ Up to reparametrization there are a unique $D_{2}$-symmetric circle and precisely two $D_{2}$-symmetric $\mathrm{tpc}_{\pi}$-curves (which are of course isometric). 
(i) Any $D_{2}$-symmetric circle $c: \mathbb{R} / \mathbb{Z} \rightarrow \mathbb{R}^{3}$ is centered at the origin and contained in the plane perpendicular to $\mathbf{e}_{2}$ with initial point $c(0) \in \mathbb{R} \mathbf{e}_{3}$.

(ii) A $D_{2}$-symmetric $\operatorname{tpc}_{\pi}: \mathbb{R} / \mathbb{Z} \rightarrow \mathbb{R}^{3}$ is located in one of the two coordinate planes $\mathbf{e}_{\mathbf{1}}^{\perp}$ and $\mathbf{e}_{\mathbf{3}}^{\perp}$. The self-intersection point is at the origin and its tangent is parallel to $\mathbf{e}_{2}$ in both cases.

Proof (i) The center $M$ of any circle $C \subset \mathbb{R}^{3}$, which - as a set - is $D_{2}$-symmetric, is the origin, since if not, we could find $i \in\{1,2,3\}$, such that $R_{i} M \neq M$ and hence $R_{i} C$ is a circle of the same length as $C$, but with a center $R_{i} M$ different from $M$. Therefore, the sets $R_{i} C$ and $C$ differ which contradicts the dihedral symmetry of $C$. Consequently, any injective arclength parametrization $c: \mathbb{R} / \mathbb{Z} \rightarrow \mathbb{R}^{3}$ of the once covered circle with $D_{2}$-symmetry satisfies the antipodal relation $c(t)=$ $-c(t+1 / 2)$ for all $t \in \mathbb{R} / \mathbb{Z}$. If there is one such parametrization $c$ with $D_{2}-$ symmetry then its image $c(\mathbb{R} / \mathbb{Z})$ must be contained in $\operatorname{span}\left\{\mathbf{e}_{\mathbf{1}}, \mathbf{e}_{\mathbf{3}}\right\}$ since, by 1-periodicity,

$$
c(t) \stackrel{(3.6)}{=} R_{2} \circ c(t-1 / 2)=R_{2} \circ c(t+1 / 2)=-R_{2} \circ c(t)
$$

for all $t \in \mathbb{R} / \mathbb{Z}$. Moreover, we infer $c(0) \in \mathbb{R} \mathbf{e}_{3}$ from (3.10) in Proposition 3.4. The existence of such a parametrization was established in Example 3.8, cf. (3.17).

(ii) For tpc $\mathrm{ta}_{\pi}$ we can argue in a similar way to see that the self-intersection point is at the origin. By Lemma 3.12 below we may assume that $\operatorname{tpc}_{\pi}$ is an arclength parametrized curve $\mathbb{R} / \mathbb{Z} \rightarrow \mathbb{R}^{3}$. Let the unit tangent of $\operatorname{tpc}_{\pi}$ at the origin be denoted by $v \in \mathbb{S}^{2}$. Due to symmetry, $\operatorname{tpc}_{\pi}\left(t+\frac{1}{2}\right)$ is just the image of a 180 degree rotation $R_{v}$ of $\operatorname{tpc}_{\pi}(t)$ about $v$. Thus we obtain

$$
R_{v} \circ \operatorname{tpc}_{\pi}(t)=\operatorname{tpc}_{\pi}\left(t+\frac{1}{2}\right) \stackrel{(3.6)}{=} R_{2} \circ \operatorname{tpc}_{\pi}(t) \quad \text { for all } t \in \mathbb{R} / \mathbb{Z} \text {. }
$$

This implies that the matrix product $R_{v}^{-1} R_{2}$ is the identity on the hyperplane which contains (the image of) tpc ${ }_{\pi}$. As it belongs to $S O(3)$, it must even be $\operatorname{Id}_{\mathbb{R}^{3}}$, in particular $v=\mathbf{e}_{2}$.

From part (i) of Proposition 3.4 we infer that the image of $\mathrm{tpc}_{\pi}$ must contain points in $\mathbb{R}_{\boldsymbol{k}} \backslash\{0\}$ for $k=1$ or $k=3$. Together with $v=\mathbf{e}_{2}$ we conclude that the planar curve tpc $\mathrm{t}_{\pi}$ belongs either to $\mathbf{e}_{\mathbf{1}}^{\perp}$ or $\mathbf{e}_{\mathbf{3}}^{\perp}$. Both configurations are realized by Example 3.9, cf. (3.18) and (3.19).

According to the following result one can reparametrize $D_{2}$-symmetric curves without affecting the symmetry. This, as well as the subsequent uniform a priori bound on the size of dihedrally symmetric curves, turns out to be useful ingredients in the existence proofs of Sect. 4.

Lemma 3.12 (Symmetry of arclength parametrization) If $\gamma \in \Sigma^{\ell}$ has length $\mathscr{L}(\gamma)=$ $L$, then its arclength parametrization $\Gamma$ is also $D_{2}$-symmetric, that is, $\Gamma$ is contained in the $D_{2}$-symmetric set $\Sigma^{L}$ defined as in (3.8). 
Proof Differentiating the symmetry relation $\gamma=\tau_{d_{i}}^{\ell}(\gamma)$ on $\mathbb{R} / \ell \mathbb{Z}$ we obtain by (3.6) and (3.2)

$$
\left|\gamma^{\prime}(t)\right|=\left|\tau_{d_{i}}^{\ell}(\gamma)^{\prime}(t)\right| \stackrel{(3.6)}{=}\left|R_{i} \circ \gamma^{\prime}\left(\psi_{i}^{\ell}(t)\right)\left(\psi_{i}^{\ell}\right)^{\prime}(t)\right| \stackrel{(3.2)}{=}\left|\gamma^{\prime}\left(\psi_{i}^{\ell}(t)\right)\right|
$$

for all $t \in \mathbb{R} / \ell \mathbb{Z}$ and $i=0,1,2,3$. This identity can be used to compute the arclength parameter

$$
\begin{aligned}
s\left(\psi_{i}^{\ell}(t)\right) & =\mathscr{L}\left(\left.\gamma\right|_{\left[0, \psi_{i}^{\ell}(t)\right]}\right)=\int_{0}^{\psi_{i}^{\ell}(t)}\left|\gamma^{\prime}(\tau)\right| d \tau \stackrel{(3.27)}{=} \int_{0}^{\psi_{i}^{\ell}(t)}\left|\gamma^{\prime}\left(\psi_{i}^{\ell}(\tau)\right)\right| d \tau \\
& =\int_{\psi_{i}^{\ell}(0)}^{t}\left|\gamma^{\prime}(z)\right| \frac{1}{\left(\psi_{i}^{\ell}\right)^{\prime}\left(\psi_{i}^{\ell}(z)\right)} d z=\left(\operatorname{sign}\left(\psi_{i}^{\ell}\right)^{\prime}\right)\left[s(t)-s\left(\psi_{i}^{\ell}(0)\right)\right]
\end{aligned}
$$

where we changed variables to $z:=\psi_{i}^{\ell}(\tau)$ with $z(0)=\psi_{i}^{\ell}(0), z\left(\psi_{i}^{\ell}(t)\right)=\psi_{i}^{\ell} \circ$ $\psi_{i}^{\ell}(t)=t$ by virtue of (3.3). Notice also that $\left(\psi_{i}^{\ell}\right)^{\prime}(\cdot)=(-1)^{i}$ for $i=0,1,2,3$. Using (3.28) with $i=2$ and $t=\ell$ we infer $2 s\left(\frac{\ell}{2}\right)=s(\ell)=L$. Now it is easy to check that

$$
\left(\operatorname{sign}\left(\psi_{i}^{\ell}\right)^{\prime}\right)\left[s(t)-s\left(\psi_{i}^{\ell}(0)\right)\right]=\psi_{i}^{L}(s(t)) \quad \text { for all } t \in \mathbb{R} / \ell \mathbb{Z}, i=0,1,2,3,
$$

where $\psi_{i}^{L}$ is the transformation defined in (3.2) only with $\ell$ replaced by $L$. In other words, $D_{2}$ acts on the domain $\mathbb{R} / L \mathbb{Z}$ of the arclength parametrization $\Gamma$ via the transformations $\psi_{i}^{L}, i=0,1,2,3$. Combining (3.28) with (3.29) we arrive at

$$
s\left(\psi_{i}^{\ell}(t)\right)=\psi_{i}^{L}(s(t)) \quad \text { for all } t \in \mathbb{R} / \ell \mathbb{Z}, i=0,1,2,3,
$$

so that the symmetry of $\gamma$ leads to

$$
\begin{gathered}
\Gamma(s(t))=\gamma(t)=\tau_{d_{i}}^{\ell}(\gamma)(t) \stackrel{(3.6)}{=} R_{i} \circ \gamma\left(\psi_{i}^{\ell}(t)\right)=R_{i} \circ \Gamma\left(s\left(\psi_{i}^{\ell}(t)\right)\right) \\
\stackrel{(3.30)}{=} R_{i} \circ \Gamma\left(\psi_{i}^{L}(s(t))\right) \stackrel{(3.6)}{=} \tau_{d_{i}}^{L}(\Gamma)(s(t))
\end{gathered}
$$

for all $t \in \mathbb{R} / \ell \mathbb{Z}, i=0,1,2,3$, which establishes the symmetry of $\Gamma$.

Lemma 3.13 (Optimal $L^{\infty}$-bound) A closed curve $\gamma \in C^{0}\left(\mathbb{R} / \ell \mathbb{Z}, \mathbb{R}^{3}\right)$ of length $\Lambda \in$ $(0, \infty)$ whose image has dihedral symmetry is contained in the closure of the ball $B_{\Lambda / 4}(0)$.

Proof Assume to the contrary that there is a point $\left(x_{1}, x_{2}, x_{3}\right)=x:=\gamma(s)$ such that $|x|>\Lambda / 4$. We may assume without loss of generality that $\left|x_{1}\right| \geq\left|x_{2}\right| \geq\left|x_{3}\right|$. The symmetry assumption means

$$
R_{1} \circ \gamma(\mathbb{R} / \ell \mathbb{Z})=R_{2} \circ \gamma(\mathbb{R} / \ell \mathbb{Z})=R_{3} \circ \gamma(\mathbb{R} / \ell \mathbb{Z})=\gamma(\mathbb{R} / \ell \mathbb{Z})
$$


so that we have $R_{i}(x) \in \gamma(\mathbb{R} / \ell \mathbb{Z})$ for $i=1,2,3$, and some permutation of the four points $A:=x, B:=R_{1}(x), C:=R_{2}(x), D:=R_{3}(x)$ forms a polygon inscribed in $\gamma$. By direct computation we infer

$$
\begin{gathered}
S:=|A-B|=|C-D|=2 \sqrt{x_{2}^{2}+x_{3}^{2}}, \\
M:=|A-C|=|B-D|=2 \sqrt{x_{1}^{2}+x_{3}^{2}}, \\
L:=|A-D|=|B-C|=2 \sqrt{x_{1}^{2}+x_{2}^{2}},
\end{gathered}
$$

with $0 \leq S \leq M \leq L$ according to our assumption on the coordinates of $x$. Of all the possible choices of permutations of the points $A, B, C, D$, the two closed polygons $P_{1}:=A B D C A$ and $P_{2}:=A C D B A$ have the shortest length $\mathscr{L}\left(P_{1}\right)=\mathscr{L}\left(P_{2}\right)=$ $2 S+2 M$, which by means of (3.33) leads to the contradictive inequality

$$
\Lambda \geq 2 S+2 M \stackrel{(3.33)}{=} 4\left(\sqrt{x_{2}^{2}+x_{3}^{2}}+\sqrt{x_{1}^{2}+x_{3}^{2}}\right) \geq 4|x|>\Lambda .
$$

This $L^{\infty}$ bound is optimal, since one can think of a sequence of ellipses of length $L$, all centered at the origin and contained in a fixed coordinate plane, converging to a straight segment of length $L / 2$ on one coordinate axis. All such ellipses are contained in the ball of radius $L / 4$ centered at the origin (cf. [32]).

Recall from the introduction the set $W_{\text {ir }}^{2,2}\left(\mathbb{R} / \ell \mathbb{Z}, \mathbb{R}^{3}\right)$ of closed, regular and embedded $W^{2,2}$-curves, each of which represents a tame knot class. Thus, for a given knot class $\mathcal{K}$ we introduce the subset

$$
\Omega_{\mathcal{K}}^{\ell}:=\left\{\gamma \in W_{\text {ir }}^{2,2}\left(\mathbb{R} / \ell \mathbb{Z}, \mathbb{R}^{3}\right):[\gamma]=\mathcal{K}\right\},
$$

which - according to the Morrey-Sobolev embedding $W^{2,2} \hookrightarrow C^{1}-$ is the empty set unless $\mathcal{K}$ is tame; see Footnote 1 . First we observe that $\Omega_{\mathcal{K}}^{\ell}$ is a Banach manifold.

Lemma $3.14\left(\Omega_{\mathcal{K}}^{\ell}\right.$ is a Banach manifold) For any fixed tame knot class $\mathcal{K}$ the set $\Omega_{\mathcal{K}}^{\ell}$ is a non-empty open subset of the Banach space $W^{2,2}\left(\mathbb{R} / \ell \mathbb{Z}, \mathbb{R}^{3}\right)$, hence a Banach manifold.

Proof By the Morrey-Sobolev embedding result any curve $\gamma \in \Omega_{\mathcal{K}}^{\ell}$ is a regular $C^{1}$-knot representing the knot class $\mathcal{K}$, so that according to Lemma 2.6 the curve $\gamma$ possesses a neighbourhood $\mathcal{U} \subset C^{1}\left(\mathbb{R} / \ell \mathbb{Z}, \mathbb{R}^{3}\right)$ such that any curve $\xi \in \mathcal{U}$ is regular and of the same knot type $\mathcal{K}$. Again by means of the Morrey-Sobolev embedding theorem we can choose the radius $\delta$ of the ball $B_{\delta}(\gamma) \subset W^{2,2}\left(\mathbb{R} / \ell \mathbb{Z}, \mathbb{R}^{3}\right)$ so small that $B_{\delta}(\gamma) \subset \mathcal{U}$, which proves the claim.

Restricting the group action (3.6) to $\Omega_{\mathcal{K}}^{\ell}$ yields a smooth $D_{2}$-manifold.

Lemma 3.15 ( $\Omega_{\mathcal{K}}^{\ell}$ is $D_{2}$-manifold) The mapping $\tau^{\ell}$ defined in (3.6) acts on the Banach manifold $\Omega_{\mathcal{K}}^{\ell}$, and under this action $\Omega_{\mathcal{K}}^{\ell}$ becomes a smooth $D_{2}$-manifold. 
Proof It is easy to see that $\tau_{d_{i}}^{\ell}(\gamma)$ is contained in $\Omega_{\mathcal{K}}^{\ell}$ for $i=0,1,2,3$, and $\gamma \in \Omega_{\mathcal{K}}^{\ell}$, since any rotation in the image and affine linear transformation of the periodic domain does not change the $W^{2,2}$-regularity and injectivity on $[0, \ell)$. Moreover, the knot class $\mathcal{K}$ is preserved as well, and

$$
\left|\tau_{d_{i}}^{\ell}(\gamma)^{\prime}(t)\right|=\left|R_{i} \circ \gamma^{\prime}\left(\psi_{i}^{\ell}(t)\right)\left(\psi_{i}^{\ell}\right)^{\prime}(t)\right|=\left|\gamma^{\prime}\left(\psi_{i}^{\ell}(t)\right)\right|>0 \text { for all } t \in \mathbb{R} / \ell \mathbb{Z} .
$$

The algebraic property (2.1) as well as the linearity of $\tau_{d_{i}}^{\ell}: \Omega_{\mathcal{K}}^{\ell} \rightarrow \Omega_{\mathcal{K}}^{\ell}$ for $i=$ $0,1,2,3$ was verified in the proof of Lemma 3.2. Indeed, the linearity of $\tau_{d_{i}}^{\ell}$ leads to the differential

$$
\left(d \tau_{d_{i}}^{\ell}\right)_{\gamma} \eta=\tau_{d_{i}}^{\ell}(\eta) \quad \text { for all } \gamma \in \Omega_{\mathcal{K}}^{\ell}, \eta \in T_{\gamma} \Omega_{\mathcal{K}}^{\ell} \simeq W^{2,2}\left(\mathbb{R} / \ell \mathbb{Z}, \mathbb{R}^{3}\right)
$$

and $i=0,1,2,3$. Therefore, $\Omega_{\mathcal{K}}^{\ell}$ is a smooth $D_{2}$-manifold, since $\tau_{d_{i}}^{\ell}: \Omega_{\mathcal{K}}^{\ell} \rightarrow \Omega_{\mathcal{K}}^{\ell}$ is a diffeomorphism with (smooth) inverse $\left(\tau_{d_{i}}^{\ell}\right)^{-1}:=\tau_{d_{i}}^{\ell}$ for each $i=0,1,2,3$ by means of the properties (3.3).

\section{Existence theory under the $D_{2}$-symmetry constraint}

Throughout this section we set $\ell=1$. Instead of the total energy $E_{\vartheta}=E+\vartheta \mathrm{TP}_{q}^{1 /(q-2)}$ which is positively (-1)-homogeneous, i.e., $E_{\vartheta}(r \gamma)=r^{-1} E_{\vartheta}(\gamma)$ for all $\gamma \in$ $W^{2,2}\left(\mathbb{R} / \mathbb{Z}, \mathbb{R}^{3}\right)$ and $r>0$, we consider the scale-invariant version

$$
S_{\vartheta}(\gamma):=\mathscr{L}(\gamma) \cdot E_{\vartheta}(\gamma)=\mathscr{L}(\gamma) \cdot\left(E(\gamma)+\vartheta \operatorname{TP}_{q}^{\frac{1}{q-2}}(\gamma)\right)
$$

We first minimize this scale-invariant total energy on the class of $W^{2,2}$-knots with dihedral symmetry, that is, we minimize $S_{\vartheta}$ on the $D_{2}$-symmetric subset

$$
\Sigma_{\mathcal{K}}:=\Sigma^{1} \cap \Omega_{\mathcal{K}}^{1}
$$

where $\Sigma^{\ell}$ for general $\ell>0$ was defined in (3.8) and $\Omega_{\mathcal{K}}^{\ell}$ in (3.34).

Theorem 4.1 (Symmetric minimizers of total scaled energy) Assume that $\Sigma_{\mathcal{K}} \neq \varnothing$ for a given knot class $\mathcal{K}$. Then for any $\vartheta>0$ there exists an arclength parametrized knot $\Gamma_{\vartheta} \in \Sigma_{\mathcal{K}}$ with length $\mathscr{L}\left(\Gamma_{\vartheta}\right)=1$, such that

$$
S_{\vartheta}\left(\Gamma_{\vartheta}\right)=\inf _{\Sigma_{\mathcal{K}}} S_{\vartheta}(\cdot)
$$

Before proving this crucial existence result, let us draw some immediate conclusions that also lead to the proofs of Theorems 1.1 and 1.3 stated in the introduction. 
Corollary 4.2 (Symmetric $S_{\vartheta}$-critical points) Any symmetric locally minimizing knot $\gamma \in \Sigma_{\mathcal{K}}$ of $\left.S_{\vartheta}\right|_{\Sigma_{\mathcal{K}}}$ is $S_{\vartheta}$-critical, that is,

$$
D S_{\vartheta}(\gamma) h=0 \quad \text { for all } h \in W^{2,2}\left(\mathbb{R} / \mathbb{Z}, \mathbb{R}^{3}\right) .
$$

Proof Small $D_{2}$-symmetric variations of a locally minimizing knot $\gamma \in \Sigma_{\mathcal{K}}$ remain regular and in the same knot class $\mathcal{K}$, so that $\gamma$ is $\left(\left.S_{\vartheta}\right|_{\Sigma_{\mathcal{K}}}\right)$-critical, that is, $D\left(\left.S_{\vartheta}\right|_{\Sigma_{\mathcal{K}}}\right)(\gamma) h=0$ for all $h \in T_{\gamma} \Sigma_{\mathcal{K}}$. Using the definition of the group action (3.6), (3.2) one easily checks that $S_{\vartheta}$ is a $D_{2}$-invariant energy. Indeed, both $E$ and $\mathrm{TP}_{q}$ are invariant under Euclidian transformations and reparametrization; here we even do not change the speed due to $\left|\left(\psi_{i}^{\ell}\right)^{\prime}(\cdot)\right|=1$ for all $i=0,1,2,3$. Furthermore, $S_{\vartheta}$ is of class $C^{1}$ by means of Theorem 2.5 .

Moreover, $\Sigma_{\mathcal{K}}$ is the non-empty $D_{2}$-symmetric subset of the smooth $D_{2}$-manifold $\Omega_{\mathcal{K}}^{1}$, cf. Lemma 3.15, so that we can apply the version of Palais's principle of symmetric criticality stated in Corollary 2.2.

Criticality of $S_{\vartheta}$ is directly related to criticality for the constrained variational problem $\left(\mathrm{lP}_{\vartheta}\right)$ for the original total energy $E_{\vartheta}$.

Corollary 4.3 (Euler-Lagrange equation) Any arclength parametrized critical point $\Gamma \in W^{2,2}\left(\mathbb{R} / \mathbb{Z}, \mathbb{R}^{3}\right)$ of $S_{\vartheta}$ satisfies

$$
D E_{\vartheta}(\Gamma)+\lambda \cdot D \mathscr{L}(\Gamma)=0
$$

for the Lagrange multiplier $\lambda:=E_{\vartheta}(\Gamma)$. Moreover, any arclength parametrized critical point for the variational problem $\left(\mathrm{P}_{\vartheta}\right)$ satisfies the same variational equation (4.5).

Proof The Euler-Lagrange equation (4.5) is a direct consequence of (4.4) via the product rule and because $\mathscr{L}(\Gamma)=1$. The variational equation for the constrained variational problem $\left(\mathrm{P}_{\vartheta}\right)$ is

$$
D E_{\vartheta}(\Gamma)+\mu \cdot D \mathscr{L}(\Gamma)=0
$$

for some Lagrange multiplier $\mu \in \mathbb{R}$, since small variations $\Gamma+\epsilon h$ for $h \epsilon$ $W^{2,2}\left(\mathbb{R} / \mathbb{Z}, \mathbb{R}^{3}\right)$ remain regular and in the same knot class $\mathcal{K}$ by Morrey's compact embedding $W^{2,2}$ into $C^{1}$. Testing (4.6) with $\Gamma$ itself we can use the fact that $\left|\Gamma^{\prime}\right| \equiv 1$ to find

$$
0=D E_{\vartheta}(\Gamma) \Gamma+\mu \int_{\mathbb{R} / \mathbb{Z}}\left|\Gamma^{\prime}(\tau)\right|^{2} d \tau=D E_{\vartheta}(\Gamma) \Gamma+\mu
$$

so that $\mu=-D E_{\vartheta}(\Gamma) \Gamma=E_{\vartheta}(\Gamma)$ by the positive (-1)-homogeneity of $E_{\vartheta}$.

Before proving Theorem 4.1 itself, we turn to another immediate application, namely the existence of symmetric critical knots for the total energy $E_{\vartheta}$ as stated in Theorem 1.1, and the existence of symmetric elastic knots; see Theorem 1.3. 
Proof of Theorem 1.1 Since by assumption there is at least one $D_{2}$-symmetric knot contained in $\mathscr{C}(\mathcal{K})$ one has $\Sigma_{\mathcal{K}} \neq \varnothing$ so that Theorem 4.1 is applicable. The $S_{\vartheta}$ minimizing knots $\Gamma_{\vartheta} \in \Sigma_{\mathcal{K}}$ obtained in that theorem have length one, so $\Gamma_{\vartheta} \in \mathscr{C}(\mathcal{K})$. They are $S_{\vartheta}$-critical according to Corollary 4.2. Moreover, $\left|\Gamma_{\vartheta}^{\prime}\right| \equiv 1$ on $\mathbb{R} / \mathbb{Z}$ so that Corollary 4.3 implies that the Euler-Lagrange equation (4.5) holds true, which is the variational equation (1.5) stated in Theorem 1.1 with the exact same Lagrange multiplier.

Proof of Theorem 1.3 For any $\vartheta>0$ we find by virtue of Theorem 4.1 an arclength parametrized knot $\Gamma_{\vartheta} \in \Sigma_{\mathcal{K}}$ (of length $\mathscr{L}\left(\Gamma_{\vartheta}\right)=1$ hence $\Gamma_{\vartheta} \in \mathscr{C}(\mathcal{K})$ ) such that

$$
E_{\vartheta}\left(\Gamma_{\vartheta}\right) \stackrel{(4.1)}{=} S_{\vartheta}\left(\Gamma_{\vartheta}\right) \stackrel{(4.3)}{\leq} S_{\vartheta}(\beta)=E_{\vartheta}(\beta)
$$

for all $D_{2}$-symmetric $\beta \in \mathscr{C}(\mathcal{K})$, since such $\beta$ are contained in $\Sigma_{\mathcal{K}}$. By definition of the total energy $E_{\vartheta}$ we infer a uniform bound on the bending energies $E\left(\Gamma_{\vartheta}\right)$,

$$
E\left(\Gamma_{\vartheta}\right) \leq E_{\vartheta}\left(\Gamma_{\vartheta}\right) \leq E_{\vartheta}(\beta) \leq E(\beta)+\mathrm{TP}_{q}^{\frac{1}{q-2}}(\beta)<\infty \text { for all } \vartheta \in(0,1]
$$

for all $D_{2}$-symmetric curves $\beta \in \mathscr{C}(\mathcal{K})$. Notice that the right-hand side is finite by virtue of Theorem 2.3. Together with the uniform $L^{\infty}$-bound $\left\|\Gamma_{\vartheta}\right\|_{L^{\infty}\left(\mathbb{R} / \mathbb{Z}, \mathbb{R}^{3}\right)} \leq 1 / 4$, which follows from Lemma 3.13 since $\mathscr{L}\left(\Gamma_{\vartheta}\right)=1$, we obtain the uniform bound

$$
\left\|\Gamma_{\vartheta}\right\|_{W^{2,2}\left(\mathbb{R} / \mathbb{Z}, \mathbb{R}^{3}\right)} \leq C<\infty \quad \text { for all } \vartheta \in(0,1]
$$

Hence, for any given sequence $\vartheta_{j} \rightarrow 0$ there exists a subsequence $\left(\vartheta_{j_{k}}\right)_{k} \subset\left(\vartheta_{j}\right)_{j}$ such that the corresponding symmetric minimizing knots $\Gamma_{\vartheta_{j_{k}}}$ converge weakly in $W^{2,2}$ and strongly in $C^{1}$ to a limiting curve $\Gamma_{0}$ as $k \rightarrow \infty$. Therefore $\Gamma_{0}$ satisfies $\left|\Gamma_{0}^{\prime}\right| \equiv 1$ on $\mathbb{R} / \mathbb{Z}, \mathscr{L}\left(\Gamma_{0}\right)=1$, the dihedral symmetry relation $\tau_{d_{i}}^{1}\left(\Gamma_{0}\right)=\Gamma_{0}$ for $i=0,1,2,3$. By the lower semicontinuity of the bending energy $E$ and by means of (4.7),

$$
E\left(\Gamma_{0}\right) \leq \liminf _{k \rightarrow \infty} E\left(\Gamma_{\vartheta_{j_{k}}}\right) \leq \liminf _{k \rightarrow \infty} E_{\vartheta_{j_{k}}}\left(\Gamma_{\vartheta_{j_{k}}}\right) \stackrel{(4.7)}{\leq} \liminf _{k \rightarrow \infty} E_{\vartheta_{j_{k}}}(\beta)=E(\beta)
$$

for all $D_{2}$-symmetric curves $\beta \in \mathscr{C}(\mathcal{K})$, which is the minimizing property (1.6).

Proof of Theorem 4.1 Since $\Sigma_{\mathcal{K}}$ was assumed to be non-empty, we have $\inf _{\Sigma_{\mathcal{K}}} S_{\vartheta} \in$ $\left[(2 \pi)^{2}, \infty\right)$, where we used that the Sobolev space $W^{2,2}$ continuously embeds ${ }^{7}$ into the fractional Sobolev space $W^{2-(1 / q), q}$ for $q \in(2,4]$ so that the tangent-point energy of a regular embedded $W^{2,2}$-curve is finite according to Theorem 2.3. Hence there exists a minimal sequence $\left(\gamma_{j}\right)_{j} \subset \Sigma_{\mathcal{K}}$ with $\lim _{j \rightarrow \infty} S_{\vartheta}\left(\gamma_{j}\right)=\inf _{\Sigma_{\mathcal{K}}} S_{\vartheta}$. Due to the scale-invariance of $S_{\vartheta}$ we may assume that $\mathscr{L}\left(\gamma_{j}\right)=1$ for all $j \in \mathbb{N}$, and we can

7 This follows, e.g., by the characterization of Sobolev spaces of real positive smoothness in terms of Triebel-Lizorkin and Besov spaces [35, Prop. 2.1.2] applied to an embedding with constant differential dimension [35, Rem. 2.2.3/2]. 
reparametrize to arclength to obtain a minimal sequence $\Gamma_{j}$ with $\left|\Gamma_{j}^{\prime}\right|=1$ for all $j$, and with

$$
\lim _{j \rightarrow \infty} E_{\vartheta}\left(\Gamma_{j}\right)=\lim _{j \rightarrow \infty} S_{\vartheta}\left(\Gamma_{j}\right)=\inf _{\Sigma_{\mathcal{K}}} S_{\vartheta}(\cdot)
$$

Note that the first equation holds since we have $\mathscr{L}\left(\Gamma_{j}\right)=1$ for all $j \in \mathbb{N}$. Moreover, $\Gamma_{j} \in \Sigma_{\mathcal{K}}$ for all $j$ due to Lemma 3.12 for $\ell=L=1$, and therefore, $\left\|\Gamma_{j}\right\|_{L^{\infty}} \leq 1 / 4$ for all $j \in \mathbb{N}$ by virtue of Lemma 3.13. Now (4.9) implies that

$$
\int_{\mathbb{R} / \mathbb{Z}}\left|\Gamma_{j}^{\prime \prime}(s)\right|^{2} d s=E\left(\Gamma_{j}\right) \leq E_{\vartheta}\left(\Gamma_{j}\right) \leq \inf _{\Sigma_{\mathcal{K}}} S_{\vartheta}(\cdot)+1<\infty \text { for all } j \gg 1,
$$

which together with the uniform $L^{\infty}$-bound and with $\left|\Gamma_{j}^{\prime}\right| \equiv 1$ for all $j$, yields a uniform bound on the full $W^{2,2}$-norm of the $\Gamma_{j}$ for $j \gg 1$. Consequently, there exists a subsequence $\left(\Gamma_{j_{k}}\right)_{k} \subset\left(\Gamma_{j}\right)_{j}$ converging weakly in $W^{2,2}$ and strongly in $C^{1}$ to a limit curve $\Gamma_{\vartheta} \in W^{2,2}\left(\mathbb{R} / \mathbb{Z}, \mathbb{R}^{3}\right)$ as $k \rightarrow \infty$. The $C^{1}$-convergence implies that $\left|\Gamma_{\vartheta}^{\prime}\right| \equiv 1$, and that $\mathscr{L}\left(\Gamma_{\vartheta}\right)=1$. Moreover, taking the limit $k \rightarrow \infty$ in the symmetry relation

$$
\tau_{d_{i}}^{1}\left(\Gamma_{j_{k}}\right)(t)=\Gamma_{j_{k}}(t) \quad \text { for all } t \in \mathbb{R} / \mathbb{Z}
$$

we find $\tau_{d_{i}}^{1}\left(\Gamma_{\vartheta}\right)=\Gamma_{\vartheta}$. To prove that $\Gamma_{\vartheta}$ is contained in $\Sigma_{\mathcal{K}}$ it suffices to show that $\Gamma_{\vartheta}$ is embedded since then $\left[\Gamma_{\vartheta}\right]=\mathcal{K}$ by Lemma 2.6. The uniform $W^{2,2}$-bound on the $\Gamma_{j_{k}}$ implies by the Morrey-Sobolev embedding also a uniform bound on the $W^{2-(1 / q), q}$-norms of the $\Gamma_{j_{k}}$. This in turn yields a uniform positive lower bound $B$ on the the bi-Lipschitz constants $\operatorname{BiLip}\left(\Gamma_{j_{k}}\right)$ according to Lemma 2.4. Passing to the limit $k \rightarrow \infty$ in the corresponding inequality

$$
\left|\Gamma_{j_{k}}(s)-\Gamma_{j_{k}}(t)\right| \geq B|s-t|_{\mathbb{R} / \mathbb{Z}} \quad \text { for all } s, t \in \mathbb{R} / \mathbb{Z}
$$

one obtains from the $C^{1}$-convergence $\Gamma_{j_{k}} \rightarrow \Gamma_{\vartheta}$

$$
\left|\Gamma_{\vartheta}(s)-\Gamma_{\vartheta}(t)\right| \geq B|s-t|_{\mathbb{R} / \mathbb{Z}} \quad \text { for all } s, t \in \mathbb{R} / \mathbb{Z}
$$

By Theorem 2.5 the tangent-point energy is lower-semicontinuous with respect to the strong $C^{1}$-convergence, and therefore also the total scaled energy $S_{\vartheta}$ with respect to the combined weak $W^{2,2}$ - and strong $C^{1}$-convergence, which implies by virtue of the fact that $\mathscr{L}\left(\Gamma_{\vartheta}\right)=1$,

$$
\inf _{\Sigma_{\mathcal{K}}} S_{\vartheta} \leq S_{\vartheta}\left(\Gamma_{\vartheta}\right)=E_{\vartheta}\left(\Gamma_{\vartheta}\right) \leq \liminf _{k \rightarrow \infty} S_{\vartheta}\left(\Gamma_{j_{k}}\right)=\inf _{\Sigma_{\mathcal{K}}} S_{\vartheta}(\cdot)
$$

We can now identify the shape of the $D_{2}$-elastic unknot as the once covered circle contained in the $\mathbf{e}_{\mathbf{1}}$-e $\mathbf{e}_{3}$-plane with starting point on the $\mathbf{e}_{\mathbf{3}}$-axis. This information is 
even more concrete than stated in Theorem 1.5, because of our choice of rotational axes in (3.6) representing the dihedral group $D_{2}$ on $\mathbb{R}^{3}$ and of the dihedral parameter transformations of the domain $\mathbb{R} / \mathbb{Z}$ described in (3.2).

Proof of Theorem 1.5 The once covered circle of length one uniquely minimizes the bending energy $E$ in $\mathscr{C}(\mathcal{K})$ where $\mathcal{K}$ is the unknot class according to the stability result by Langer and Singer [29]. But it also uniquely minimizes the tangent-point energy $\mathrm{TP}_{q}$ by the two uniqueness proofs of Volkmann and Blatt; see [39, Cor. 5.12]. Therefore the once covered circle of length one and all its isometric images also uniquely minimize the total energy $E_{\vartheta}$ within $\mathscr{C}(\mathcal{K})$ for any $\vartheta>0$. The dihedral symmetry forces the $E_{\vartheta}$-minimizing circles to lie in the $\mathbf{e}_{\mathbf{1}}-\mathbf{e}_{\mathbf{3}}$-plane, with initial point contained in $\mathbb{R e}_{3}$; see Corollary 3.11. These properties transfer via $C^{1}$-convergence of $E_{\vartheta_{j}}$-minimizers as $\vartheta_{j} \rightarrow 0$ to the elastic unknot.

Now assume that a $D_{2}$-elastic knot for some knot class $\mathcal{K}$ is the once covered circle, which represents the unknot class. According to Lemma 2.6 there is an entire $C^{1}$-neighborhood of the once covered circle that only consists of unknots. But by definition of elastic knots this once covered circle is the $C^{1}$-limit of $E_{\vartheta_{j}}$-minimizing knots $\Gamma_{\vartheta_{j}}, \vartheta_{j} \rightarrow 0$, all representing the knot class $\mathcal{K}$. This implies that $\mathcal{K}$ is the unknot.

Notice finally, for the proof of (1.7), that Fenchel's lower bound of $2 \pi$ for the total curvature of any closed curve combined with Hölder's inequality implies that $(2 \pi)^{2}$ is the infimal bending energy for the trivial knot class. This value is attained by the once covered circle. Now (1.7) immediately follows from the fact that according to Corollary 3.11 there are round $D_{2}$-symmetric circles.

Now we turn to non-trivial knot classes satisfying assumption (1.8) on the infimal bending energy. Here we need [24, Theorem A.1] where the Fáry-Milnor theorem on the lower bound for total curvature of non-trivially knotted curves has been extended to the $C^{1}$-closure of knots. We restate it for the convenience of the reader.

Theorem 4.4 (Fáry-Milnor extension) Let $\mathcal{K}$ be a non-trivial (tame) knot class and suppose $\gamma$ belongs to the $C^{1}$-closure of $\mathscr{C}(\mathcal{K})$. Then $\mathrm{TC}(\gamma) \geq 4 \pi$.

This result permits to prove the following rigidity result, which is the essential ingredient for the proof of Theorem 1.6 presented in Section 5.

Theorem 4.5 (Rigidity \& strong convergence) If a knot class $\mathcal{K}$ satisfies (1.8) then any $D_{2}$-elastic knot $\Gamma_{0}$ for $\mathcal{K}$ is (up to reparametrization) the tangential pair of co-planar circles with exactly one point in common described in Corollary 3.11. In addition, any subsequence of $D_{2}$-symmetric $E_{\vartheta}$-minimizers $\Gamma_{\vartheta} \in \mathscr{C}(\mathcal{K})$ converges strongly in $W^{2,2}$ to (an isometric image of) $\Gamma_{0}$ as $\vartheta \rightarrow 0$.

Proof From Theorem 1.5 we infer that $\mathcal{K}$ is nontrivial. Applying the extended FáryMilnor Theorem 4.4 to any $D_{2}$-elastic knot $\Gamma_{0}$ which according to Theorem 1.3 lies in the $C^{1}$-closure of $\mathscr{C}(\mathcal{K})$, we estimate by means of Hölder's inequality

$$
(4 \pi)^{2} \stackrel{\text { Thm. }}{\leq}{ }^{4.4}\left(\int_{\Gamma_{0}} \kappa_{\Gamma_{0}} d s\right)^{2} \leq E\left(\Gamma_{0}\right) \stackrel{(1.6)}{\leq} \inf _{\substack{\beta \in \mathscr{C}(\mathcal{K}) \\ \beta D_{2} \text {-symmetric }}} E(\beta) \stackrel{(1.8)}{=}(4 \pi)^{2}
$$


Consequently, we have equality everywhere, in particular equality in Hölder's inequality, which implies a constant integrand $\kappa_{\Gamma_{0}}=4 \pi$ a.e. on $\mathbb{R} / \mathbb{Z}$.

Next we prove that $\Gamma_{0}$ has at least one double point. Indeed, otherwise, by Lemma 2.6 the curve $\Gamma_{0}$ would be contained in $\mathscr{C}(\mathcal{K})$ since $\Gamma_{0}$ is the strong $C^{1}$-limit of the $E_{\vartheta_{j}}$-minimizers $\Gamma_{\vartheta_{j}} \in \mathscr{C}(\mathcal{K})$ as $\vartheta_{j} \rightarrow 0$. Combining the minimizing property (1.6) of $\Gamma_{0}$ with our assumption (1.8), which also implies that $(4 \pi)^{2}=\inf _{\mathscr{C}(\mathcal{K})} E$, we find that $\Gamma_{0}$ is an embedded minimizer of the bending energy within $\mathscr{C}(\mathcal{K})$, hence a stable critical point of $E$. Applying the stability result of Langer and Singer [29], we find $\Gamma_{0}$ to be the once covered circle representing the unknot class, which contradicts the fact that $\mathcal{K}$ is nontrivial. So, we have shown that $\Gamma_{0}$ is not injective.

According to [24, Cor. 3.4] the elastic $D_{2}$-symmetric knot $\Gamma_{0}$ belongs, up to isometry and reparametrization, to the one-parameter family of tangentially intersecting circles $\operatorname{tpc}_{\varphi}$ for $\varphi \in[0, \pi]$ explicitly given in [24, Formula (3.2)]. One easily checks that the only possible candidates that may respect the $D_{2}$-symmetry are the doubly covered circle $\mathrm{tpc}_{0}$ and the tangential pair of co-planar circles $\mathrm{tpc}_{\pi}$ with only one touching point.

But (any isometric image of) $\mathrm{tpc}_{0}$ is not only 1-periodic but also 1/2-periodic, so that the symmetry assumption $\tau_{d_{2}}^{1}\left(\operatorname{tpc}_{0}\right)=\operatorname{tpc}_{0}$ would lead to

$$
\begin{gathered}
\operatorname{tpc}_{0}(t)=\operatorname{tpc}_{0}(t+1 / 2)=\tau_{d_{2}}^{1}\left(\operatorname{tpc}_{0}\right)(t+1 / 2) \stackrel{(3.6)}{=} R_{2} \circ \operatorname{tpc}_{0}\left(\psi_{2}^{1}(t+1 / 2)\right) \\
\stackrel{(3.2)}{=} R_{2} \circ \operatorname{tpc}_{0}(t) \text { for all } t \in \mathbb{R} / \mathbb{Z},
\end{gathered}
$$

So that $\operatorname{tpc}_{0}(t) \in \mathbb{R} \mathbf{e}_{2}$ for all $t \in \mathbb{R} / \mathbb{Z}$, which is a contradiction.

So, (up to isometry) the only remaining option is $\Gamma_{0}=\operatorname{tpc}_{\pi}$, and that this curve indeed has the $D_{2}$-symmetry has been verified in Example 3.9.

It remains to establish strong convergence. Now that we have identified the weak $W^{2,2}$-limit of the $E_{\vartheta_{j}}$-minimizers for any sequence $\vartheta_{j} \rightarrow 0$, we can use our assumption (1.8) to find for any given $\delta>0$ a $D_{2}$-symmetric curve $\beta \in \mathscr{C}(\mathcal{K})$ such that $E(\beta) \leq(4 \pi)^{2}+\delta$. The minimizing property of the $\Gamma_{\vartheta}$ yields

$$
(4 \pi)^{2} \leq E\left(\Gamma_{\vartheta}\right) \leq E_{\vartheta}\left(\Gamma_{\vartheta}\right) \leq E_{\vartheta}(\beta) \leq(4 \pi)^{2}+\delta+\vartheta \operatorname{TP}_{q}(\beta)^{\frac{1}{q-2}}<\infty,
$$

where we used the classic Fáry-Milnor theorem for the first inequality. Taking the limit $\vartheta \rightarrow 0$ gives

$$
(4 \pi)^{2} \leq \liminf _{\vartheta \rightarrow 0} E\left(\Gamma_{\vartheta}\right) \leq \limsup _{\vartheta \rightarrow 0} E\left(\Gamma_{\vartheta}\right) \leq(4 \pi)^{2}+\delta \text { for all } \delta>0 .
$$

Therefore, $\lim _{\vartheta \rightarrow 0} E\left(\Gamma_{\vartheta}\right)=(4 \pi)^{2}=E\left(\Gamma_{0}\right)$, since $\kappa_{\Gamma_{0}}=4 \pi$ a.e. on $\mathbb{R} / \mathbb{Z}$. This, together with the $C^{1}$-convergence of the $\Gamma_{\vartheta}$ to the same limit (up to permutation of the axes and reparametrization) leads to convergence in the $W^{2,2}$-norm. Combining this with the weak convergence to the now unique weak limit $\Gamma_{0}$ (up to permutation of the axes and reparametrization) gives finally strong convergence in $W^{2,2}$ by the subsequence principle. 


\section{Infimal bending energy on torus knots with dihedral symmetry}

We now investigate the convergence properties of the $D_{2}$-symmetric torus knots $g_{3}^{\epsilon}$ introduced in Example 3.10 fixing $\ell:=1$ and $\varrho:=\epsilon^{2}$.

Lemma 5.1 ( $W^{2,2}$-convergence of $D_{2}$-symmetric torus knots) Let $\ell=1, \varrho=\epsilon^{2}$, and $b \in \mathbb{Z} \backslash\{1,-1\}$ be odd. Then the $D_{2}$-symmetric torus knots $g_{3}^{\epsilon} \in \Sigma_{\mathcal{T}(2, b)}$ constructed in Example 3.10 for $\ell=1$ and $\varrho=\epsilon^{2}$ converge strongly in $W^{2,2}$ to the tangential pair of co-planar circles $\mathrm{tpc}_{\pi}$ (see Example 3.9) as $\epsilon \rightarrow 0$.

Corollary 5.2 (Minimal bending energy for $D_{2}$-symmetric torus knots) The torus knot classes $\mathcal{T}(2, b)$ for odd $b \in \mathbb{Z} \backslash\{1,-1\}$ are the only knot classes $\mathcal{K}$ that satisfy

$$
\inf _{\substack{\beta \in \mathscr{C}(\mathcal{K}) \\ \beta D_{2} \text {-symmetric }}} E(\beta)=(4 \pi)^{2}=\inf _{\mathscr{C}(\mathcal{K})} E(\cdot) .
$$

Proof The convergence of torus knots established in Lemma 5.1 together with an appropriate rescaling to unit length, i.e., the $W^{2,2}$-convergence of $g_{3}^{\epsilon} / \mathscr{L}\left(g_{3}^{\epsilon}\right) \in$ $\Sigma_{\mathcal{T}(2, b)}$ to tpc $\mathrm{c}_{\pi}$, allows us to identify the infimal bending energy $(4 \pi)^{2}=E\left(\operatorname{tpc}_{\pi}\right)$ on the class of $D_{2}$-symmetric curves in $\mathscr{C}(\mathcal{T}(2, b))$, since by the Morrey-Sobolev embedding we have $g_{3}^{\epsilon} \rightarrow \operatorname{tpc}_{\pi}$ in $C^{1}$, and hence $\mathscr{L}\left(g_{3}^{\varepsilon}\right) \rightarrow \mathscr{L}\left(\mathrm{tpc}_{\pi}\right)=1$, and therefore also $g_{3}^{\epsilon} / \mathscr{L}\left(g_{3}^{\epsilon}\right) \rightarrow \operatorname{tpc}_{\pi}$ in $W^{2,2}$ as $\epsilon \rightarrow 0$. That the torus knot classes $\mathcal{T}(2, b)$ are the only possible knot classes to satisfy the right equality in (1.8) was proven in [24, Corollary 4.4].

Proof of Lemma 5.1 For simplicity we restrict the explicit arguments to the case that $b \geq 3$. By symmetry it suffices to prove the $W^{2,2}$-convergence of the generating arcs $\alpha^{\epsilon}:=\alpha_{3}^{\epsilon}$ of $g_{3}^{\epsilon}$ to the corresponding generating arc $\alpha_{2}$ of $\mathrm{tpc}_{\pi}$ defined in Example 3.9. Since $\alpha_{3}^{\epsilon}$ is piecewise defined (see (3.25)) we focus first on the interval $I_{1}(\epsilon):=$ $[0,(b+1) \epsilon / 2]$ where $\alpha_{3}^{\epsilon}=h^{\epsilon}$, and obtain by direct computation from the explicit expressions (3.20) and (3.21) for the helical part $h^{\epsilon}$ and the parameter transformation $\phi\left(\right.$ for $\left.\varrho=\epsilon^{2}\right)$

$$
\left|\left(h^{\epsilon}\right)^{\prime \prime}(t)\right|^{2}=\pi^{4} \phi^{\prime 4}(t / \epsilon)+\pi^{2} \phi^{\prime \prime 2}(t / \epsilon) \quad \text { for all } t \in I_{1}(\epsilon),
$$

which implies

$$
\left\|\left(h^{\epsilon}\right)^{\prime \prime}\right\|_{L^{2}\left(I_{1}(\epsilon), \mathbb{R}^{3}\right)}^{2} \leq \epsilon \pi^{2} \int_{0}^{(b+1) / 2}\left(\pi^{2} \phi^{\prime 4}(z)+1\right) d z,
$$

where we changed variables to $z:=t / \epsilon$ and used that $\left|\phi^{\prime \prime}\right| \leq 1$ on $[0,(b+1) / 2)$. Now, $\phi^{\prime}=1$ on $[0,(b-1) / 2]$ whereas $\phi^{\prime}(t)=-(t-(b+1) / 2)$ for $t \in[(b-1) / 2,(b+1) / 2]$ according to (3.21) so that we obtain from (5.2)

$$
\left\|\left(h^{\epsilon}\right)^{\prime \prime}\right\|_{L^{2}\left(I_{1}(\epsilon), \mathbb{R}^{3}\right)}^{2} \leq \epsilon \pi^{2}\left[\pi^{2}\left(\frac{b-1}{2}+\frac{1}{5}\right)+\frac{b+1}{2}\right]<\epsilon \pi^{4}(b+1) .
$$


The prospective arclength parametrized limit curve $\operatorname{tpc}_{\pi}$ has constant curvature $\left|\operatorname{tpc}_{\pi}^{\prime \prime}\right|=4 \pi$ a.e., so that by virtue of (5.3)

$$
\left\|\left(\alpha^{\epsilon}\right)^{\prime \prime}-\alpha_{2}^{\prime \prime}\right\|_{L^{2}\left(I_{1}(\epsilon), \mathbb{R}^{3}\right)}^{2} \stackrel{(5.3)}{\leq} 2 \epsilon \pi^{4}(b+1)+(4 \pi)^{2}(b+1) \epsilon .
$$

Now we consider the interval $I_{2}(\epsilon):=[(b+1) \epsilon / 2,(1 / 4)-(b+1) \epsilon / 2]$ and recall our condition (3.23) on the radius $r$ of the stadium curve $\sigma^{\epsilon}$, namely (now for $\ell=1$ )

$$
(b+1) \epsilon+\pi r=1 / 4 \text {. }
$$

This implies that the auxiliary function $f(t, s):=\left|r^{-1}(t-(b+1) s / 2)-4 \pi t\right|^{2}$ satisfies for any $\epsilon \in\left(0,(8(b+1))^{-1}\right)$

$$
0 \leq f(t, s)<1024 \pi^{2}(b+1)^{2} \epsilon^{2} \text { for all }(t, s) \in[0,1] \times[0,2 \epsilon]
$$

since $\sqrt{f(t, s)}<4 \pi(b+1)|8 \varepsilon t-s| \leq 4 \pi(b+1) \max (8 \varepsilon, 2 \varepsilon) \leq 32 \pi(b+1) \varepsilon$. Inequality (5.6) can be used to estimate

$$
\begin{aligned}
&\left\|\left(\alpha^{\epsilon}\right)^{\prime \prime}-\alpha_{2}^{\prime \prime}\right\|_{L^{2}\left(I_{2}(\epsilon), \mathbb{R}^{3}\right)}^{2} \leq 2 \int_{I_{2}(\epsilon)}|(1 / r)-4 \pi|^{2} d t \\
&+2 \cdot(4 \pi)^{2} \int_{I_{2}(\epsilon)}\left|\left(\begin{array}{c}
\cos ((t-(b+1) \epsilon / 2) / r)-\cos (4 \pi t) \\
-\sin ((t-(b+1) \epsilon / 2) / r)+\sin (4 \pi t) \\
0
\end{array}\right)\right|^{2} d t
\end{aligned}
$$

The first integrand equals $f(1,0)$, and the second integrand can be estimated from above by $2 f(t, \epsilon)$ for $t \in I_{2}(\epsilon)$, since both, cos and sin, have Lipschitz constant 1 . Therefore, we can apply the auxiliary estimate (5.6) to arrive at

$$
\left\|\left(\alpha^{\epsilon}\right)^{\prime \prime}-\alpha_{2}^{\prime \prime}\right\|_{L^{2}\left(I_{2}(\epsilon), \mathbb{R}^{3}\right)}^{2}<\frac{1}{2}\left(1+2(4 \pi)^{2}\right) 1024 \pi^{2}(b+1)^{2} \epsilon^{2},
$$

where we also used that $\mathscr{L}^{1}\left(I_{2}(\epsilon)\right)<1 / 4$. Finally, on the interval $I_{3}(\epsilon):=[(1 / 4)-$ $(b+1) \epsilon / 2,1 / 4]$ the stadium curve $\left.\sigma\right|_{I_{3}(\epsilon)}=\alpha^{\epsilon}$ is a straight segment so that

$$
\left\|\left(\alpha^{\epsilon}\right)^{\prime \prime}-\alpha_{2}^{\prime \prime}\right\|_{L^{2}\left(I_{3}(\epsilon), \mathbb{R}^{3}\right)}^{2}=\left\|\alpha_{2}^{\prime \prime}\right\|_{L^{2}\left(I_{3}(\epsilon), \mathbb{R}^{3}\right)}^{2}=(4 \pi)^{2}(b+1) \epsilon / 2 .
$$

Summarizing (5.4), (5.7), and (5.8) we obtain a constant $C_{1} \geq 1$ independent of $\epsilon$ such that

$$
\left\|\left(\alpha^{\epsilon}\right)^{\prime \prime}-\alpha_{2}^{\prime \prime}\right\|_{L^{2}\left([0,1 / 4], \mathbb{R}^{3}\right)} \leq C_{1} \sqrt{\epsilon} \quad \text { for all } 0<\epsilon<\frac{1}{8(b+1)},
$$

which by means of Poincaré's inequality [20, Section 5.8.1] applied to $\gamma^{\prime}$ (satisfying $\int_{\mathbb{R} / \mathbb{Z}} \gamma^{\prime}(\tau) d \tau=0$ because $\gamma$ is 1 -periodic) implies that there is a constant $C_{2} \geq 1$ such that 


$$
\left\|\left(\alpha^{\epsilon}\right)^{\prime}-\alpha_{2}^{\prime}\right\|_{W^{1,2}\left([0,1 / 4], \mathbb{R}^{3}\right)} \leq C_{2} \sqrt{\epsilon} \quad \text { for all } 0<\epsilon<\frac{1}{8(b+1)}
$$

To conclude the proof it therefore suffices to prove the uniform convergence of the $\alpha^{\epsilon}$ to $\alpha_{2}$ on $[0,1 / 4]$ as $\epsilon \rightarrow 0$. We have for any $t \in\left[0, \frac{1}{4}\right]$

$$
\begin{aligned}
\left|\alpha^{\epsilon}(t)-\alpha_{2}(t)\right| & =\left|\alpha^{\epsilon}(0)-\alpha_{2}(0)\right|+\left|\int_{0}^{t}\left(\left(\alpha^{\epsilon}\right)^{\prime}-\alpha_{2}^{\prime}\right)\right| \\
& \leq \varrho+\sqrt{t}\left\|\left(\alpha^{\epsilon}\right)^{\prime}-\alpha_{2}^{\prime}\right\|_{L^{2}} \leq \epsilon^{2}+\frac{1}{2} C_{2} \sqrt{\epsilon} .
\end{aligned}
$$

Taking the supremum over $t \in\left[0, \frac{1}{4}\right]$ concludes the proof.

Proof of Theorem 1.6 (i) Corollary 5.2 implies that the torus knot classes $\mathcal{T}(2, b)$ for odd $b \in \mathbb{Z} \backslash\{1,-1\}$ satisfy condition (1.8), so that Theorem 4.5 is applicable.

(ii) If a $D_{2}$-symmetric elastic knot for some knot class $\mathcal{K}$ is $\operatorname{tpc}_{\varphi}$ then there is a sequence of curves $\left(\gamma_{k}\right)_{k \in \mathbb{N}} \subset \mathscr{C}(\mathcal{K})$ such that $\gamma_{k} \rightarrow \operatorname{tpc}_{\pi}$ with respect to the $C^{1}$-norm. For all $\gamma_{k}$ sufficiently close to $\operatorname{tpc}_{\pi}$ with respect to the $C^{1}$-norm, we obtain some cumulative angle $\Delta \beta$ as described in [24, Prop. 4.2] which yields the existence of some odd integer $b$ such that $\gamma_{k}$ is a $(2, b)$-torus knot if $|b| \geq 3$ and unknotted if $b= \pm 1$. The latter is ruled out by Theorem 1.5 .

(iii) This is an immediate consequence of Corollary 5.2.

\section{Discussion and open problems}

\subsection{Higher regularity of $D_{2}$-symmetric $E_{\vartheta}$-critical knots}

The Euler-Lagrange operator of $\mathrm{TP}_{q}$ for $q>2$ studied in [10] seems to be related to the $q$-Laplacian for which one cannot expect full regularity, at least in the nonfractional case. So it is open whether the arclength parametrized $D_{2}$-symmetric critical points of the constrained variational problem $\left(\mathrm{P}_{\vartheta}\right)$ obtained in Theorem 1.1 are of class $C^{\infty}\left(\mathbb{R} / \mathbb{Z}, \mathbb{R}^{3}\right)$.

Choosing instead of $\mathrm{TP}_{q}$ the decoupled tangent-point functionals $\mathrm{TP}^{(p, 2)}$ for $p \in(4,5)$, cf. Footnote 4 , whose domain is a Hilbert space, we can generalize the bootstrapping argument from [10] to obtain $C^{\infty}$-regularity. We might even derive analyticity by extending the arguments given in $[12,36,41]$.

\subsection{Non-embeddedness of symmetric elastic knots}

Similarly as in [24, Proposition 3.1] we expect that also symmetric elastic knots for non-trivial knot classes must have double points. According to the stability result of Langer and Singer [29], the only stable critical point of the bending energy $E$ is the once covered circle. However, due to the fact that the symmetry constraint only permits to apply symmetry preserving variations in (1.6), we cannot immediately apply this 
tool in the present work. Consequently, in contrast to the case of (not necessarily symmetric) elastic knots treated in [24], we are presently not able to show that

- every $D_{2}$-elastic knot for a non-trivial knot class $\mathcal{K}$ must have self-intersection points, and that

- inequality (1.6) is strict unless $\mathcal{K}$ is the unknot class.

It is an interesting question whether one may derive a weaker version of the stability result in [29], that is applicable in our situation, e.g., stating that the round circle would be the only local minimizer within the $D_{2}$-symmetric subclass $\Sigma^{1}$ introduced in Definition 3.3. In this case, one could argue as in [24] to conclude that any embedded minimizer of the bending energy within the set $\Sigma_{\mathcal{K}}$ of $D_{2}$-symmetric knots defined in (4.2) would in fact be a local minimizer within $\Sigma^{1}$. Hence, $\mathcal{K}$ would be the unknot contradicting the assumption of a non-trivial knot class $\mathcal{K}$. Thus, the $D_{2}$-symmetric minimizer of the bending energy could not be embedded, and the infimum of the bending energy could not be attained in $\Sigma_{\mathcal{K}}$.

\subsection{Other knot classes and symmetries}

In a similar manner as in Definition 1.4 we may define $G$-elastic knots for any symmetry group $G$. Most results from the Introduction, namely Theorems 1.1, 1.3, and 1.5 as well as Corollary 1.2, carry over to more general symmetry groups while Theorem 1.6 is restricted to $D_{2}$. We briefly speculate about some examples involving other knot classes or symmetry groups different from $D_{2}$.

\section{General torus knots}

Let $a, b \in \mathbb{N}$ be coprime with $2 \leq a<b$. We expect the elastic knot to be the $a$-times covered circle [24] which then would agree with the $D_{b}$-elastic knot.

Consequently, the $D_{a}$-elastic knot is likely to be the union of $a$ circles of radius $1 /(2 \pi a)$ that tangentially meet in one common point. The angle between two consecutive circles amounts to $2 \pi / a$, which is also observed experimentally by means of the numerical gradient flow of Bartels et al.; see Fig. 5A. An animation is available as an ancillary file at arXiv:2105.08558v2.

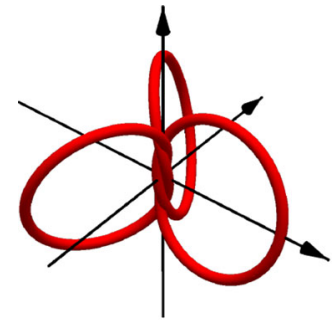

(A)

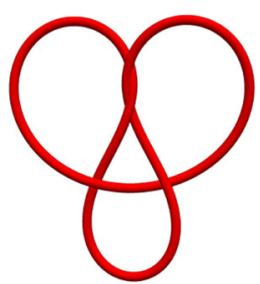

(B)

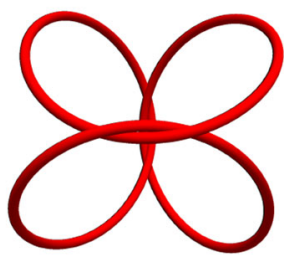

(C)

Fig. 5 Numerical approximations of candidates for $\mathbf{A}$ the $D_{3}$-elastic $(3,4)$-torus knot, $\mathbf{B}$ the elastic figureeight knot, $\mathbf{C}$ the $D_{2}$-elastic figure-eight knot 


\section{The figure-eight knot}

Simulations that have been carried out earlier suggest that an elastic figure eight $\left(4_{1}\right)$ is either planar [3] or spherical [23,24]; see Fig. 5B, C. Recent numerical experiments [6] support the former. So the planar configuration (B) could be a global minimizer within the figure-eight class whereas the spherical configuration (C) might merely be a local minimizer.

Assuming that this is true and that there are no further candidates, we may conjecture that the planar configuration is an elastic knot, while the spherical configuration is a $D_{2}$-elastic knot. In contrast to the latter, the former does not enjoy a $D_{2}$-symmetry. Using the theory developed above, we can only state that there exists a $D_{2}$-elastic figure-eight knot which may or may not coincide with the elastic figure-eight.

Acknowledgements We are indebted to Sören Bartels for fruitful discussions on the numerical approximation of elastic knots. While working on this paper, the first author was an International Research Fellow of JSPS (Postdoctoral Fellowships for Research in Japan). The second author has been partially supported by Grant RE 3930/1-1 of the German Research Foundation (DFG). The third author's work is partially funded by DFG Grant no. Mo 966/7-1 Geometric curvature functionals: energy landscape and discrete methods (project no. 282535003), and by the Excellence Initiative of the German federal and state governments. A meeting of all three authors in 2020 at RWTH Aachen University was generously funded by the DFGGraduiertenkolleg Energy, Entropy, and Dissipative Dynamics (EDDy), project no. 320021702/GRK2326.

Funding Please refer to the Acknowledgments.

Availability of data and material Data sharing not applicable to this article as no datasets were generated or analysed during the current study.

\section{Declarations}

Conflict of interest The author declares that they have no conflict of interest.

Code availability Details on the code that has been used for the simulations in Sect. 6 can be found in [6].

Open Access This article is licensed under a Creative Commons Attribution 4.0 International License, which permits use, sharing, adaptation, distribution and reproduction in any medium or format, as long as you give appropriate credit to the original author(s) and the source, provide a link to the Creative Commons licence, and indicate if changes were made. The images or other third party material in this article are included in the article's Creative Commons licence, unless indicated otherwise in a credit line to the material. If material is not included in the article's Creative Commons licence and your intended use is not permitted by statutory regulation or exceeds the permitted use, you will need to obtain permission directly from the copyright holder. To view a copy of this licence, visit http://creativecommons.org/licenses/by/4.0/.

\section{References}

1. Adams, C.C.: The knot book. American Mathematical Society, Providence (2004)

2. Alt, H.W.: Linear Functional Analysis. Springer, London (2016). https://doi.org/10.1007/978-1-44717280-2 (Translated by R. Nürnberg)

3. Avvakumov, S., Sossinsky, A.: On the normal form of knots. Russ. J. Math. Phys. 21(4), 421-429 (2014). https://doi.org/10.1134/S1061920814040013 
4. Bartels, S.: A simple scheme for the approximation of the elastic flow of inextensible curves. IMA J. Numer. Anal. 33(4), 1115-1125 (2013). https://doi.org/10.1093/imanum/drs041

5. Bartels, S., Falk, Ph., Weyer, P.: KNOTevolve-a tool for relaxing knots and inextensible curves. Web application (2020). https://aam.uni-freiburg.de/agba/forschung/knotevolve/

6. Bartels, S., Reiter, Ph.: Stability of a simple scheme for the approximation of elastic knots and selfavoiding inextensible curves. Math. Comput. 90(330), 1499-1526 (2021). https://doi.org/10.1090/ $\mathrm{mcom} / 3633$

7. Bartels, S., Reiter, Ph., Riege, J.: A simple scheme for the approximation of self-avoiding inextensible curves. IMA J. Numer. Anal. 38(2), 543-565 (2018). https://doi.org/10.1093/imanum/drx021

8. Blatt, S.: Note on continuously differentiable isotopies. Report 34, Institute for Mathematics, RWTH Aachen, (2009). http://www.instmath.rwth-aachen.de/Preprints/blatt20090825.pdf

9. Blatt, S.: The energy spaces of the tangent-point energies. J. Topol. Anal. 5(3), 261-270 (2013)

10. Blatt, S., Reiter, Ph.: Regularity theory for tangent-point energies: the non-degenerate sub-critical case. Adv. Calc. Var. 8(2), 93-116 (2015). https://doi.org/10.1515/acv-2013-0020

11. Blatt, S., Reiter, Ph.: Towards a regularity theory for integral Menger curvature. Ann. Acad. Sci. Fenn. Math. 40(1), 149-181 (2015). https://doi.org/10.5186/aasfm.2015.4006

12. Blatt, S., Vorderobermeier, N.: On the analyticity of critical points of the Möbius energy. Calc. Var. Partial Differ. Equ. 58(1), 28 (2019). https://doi.org/10.1007/s00526-018-1443-6 (Paper No. 16)

13. Buck, G., Orloff, J.: A simple energy function for knots. Topol. Appl. 61(3), 205-214 (1995). https:// doi.org/10.1016/0166-8641(94)00024-W

14. Burde, G., Zieschang, H.: Knots, volume 5 de Gruyter Studies in Mathematics, 2nd edn. Walter de Gruyter \& Co., Berlin (2003)

15. Cantarella, J., Fu, J.H.G., Kusner, R.B., Sullivan, J.M.: Ropelength criticality. Geom. Topol. 18(4), 1973-2043 (2014). https://doi.org/10.2140/gt.2014.18.1973

16. Cohn, P.M.: Lie Groups. Cambridge Tracts in Mathematics and Mathematical Physics, no. 46. Cambridge University Press, New York (1957)

17. Crowell, R.H., Fox, R.H.: Introduction to knot theory. Springer, New York (1977). https://doi.org/10. 1007/978-1-4612-9935-6 (Reprint of the 1963 original, Graduate Texts in Mathematics, No. 57)

18. Denne, E., Sullivan, J.M.: Convergence and isotopy type for graphs of finite total curvature. In: Discrete differential geometry, volume 38 of Oberwolfach Semin, pp. 163-174. Birkhäuser, Basel (2008). https://doi.org/10.1007/978-3-7643-8621-4_8

19. Diao, Y., Ernst, C., Janse van Rensburg, E.J.: Thicknesses of knots. Math. Proc. Cambridge Philos. Soc. 126(2), 293-310 (1999). https://doi.org/10.1017/S0305004198003338

20. Evans, L.C.: Partial Differential Equations. Graduate Studies in Mathematics, vol. 19. American Mathematical Society, Providence (1998)

21. Fáry, I.: Sur la courbure totale d'une courbe gauche faisant un nœud. Bull. Soc. Math. France, 77:128138, (1949). http://www.numdam.org/item?id=BSMF_1949_77_128_0

22. Fenchel, W.: Über Krümmung und Windung geschlossener Raumkurven. Math. Ann. 101(1), 238-252 (1929). https://doi.org/10.1007/BF01454836

23. Gallotti, R., Pierre-Louis, O.: Stiff knots. Phys. Rev. E (3) 75(3), 03180, 14 (2007). https://doi.org/10. 1103/PhysRevE.75.031801

24. Gerlach, H., Reiter, Ph., von der Mosel, H.: The elastic trefoil is the doubly covered circle. Arch. Ration. Mech. Anal. 225(1), 89-139 (2017). https://doi.org/10.1007/s00205-017-1100-9

25. Gilsbach, A.: On symmetric critical points of knot energies. PhD thesis, RWTH Aachen University (2018). http://publications.rwth-aachen.de/record/726186/files/726186.pdf

26. Gilsbach, A., von der Mosel, H.: Symmetric critical knots for O'Hara's energies. Topol. Appl. 242, 73-102 (2018). https://doi.org/10.1016/j.topol.2018.04.014. Update on arXiv:1709.06949

27. Gonzalez, O., Maddocks, J.H.: Global curvature, thickness, and the ideal shapes of knots. Proc. Natl. Acad. Sci. USA 96(9), 4769-4773 (1999). https://doi.org/10.1073/pnas.96.9.4769 (electronic)

28. Grünbaum, B., Shephard, G.: Symmetry groups of knots. Math. Mag. 58(3), 161-165 (1985)

29. Langer, J., Singer, D.A.: Curve straightening and a minimax argument for closed elastic curves. Topology 24(1), 75-88 (1985). https://doi.org/10.1016/0040-9383(85)90046-1

30. Milnor, J.W.: On the total curvature of knots. Ann. Math. 2(52), 248-257 (1950). https://doi.org/10. 2307/1969467

31. Natanson, I.P., Boron, L.F.: Theory of Functions of a Real Variable. Courier Dover Publications, New York (2016) 
32. Nitsche, J.C.C.: The smallest sphere containing a rectifiable curve. Am. Math. Monthly 78, 881-882 (1971). https://doi.org/10.2307/2316484

33. Palais, R.S.: The principle of symmetric criticality. Commun. Math. Phys. 69(1), 19-30 (1979). http:// projecteuclid.org/euclid.cmp/1103905401

34. Reiter, Ph.: All curves in a $C^{1}$-neighbourhood of a given embedded curve are isotopic. Report 4 , Institute for Mathematics, RWTH Aachen, (2005). http://www.instmath.rwth-aachen.de/Preprints/ reiter20051017.pdf

35. Runst, T., Sickel, W.: Sobolev Spaces of Fractional Order, Nemytskij operators, and Nonlinear Partial Differential Equations, volume 3 of De Gruyter Series in Nonlinear Analysis and Applications. Walter de Gruyter \& Co., Berlin (1996). https://doi.org/10.1515/9783110812411

36. Steenebrügge, D., Vorderobermeier, N.: On the analyticity of critical points of the generalized Integral Menger Curvature in the Hilbert case. arXiv e-prints, (2021). arXiv:2103.07383

37. Strzelecki, P., Szumańska, M., von der Mosel, H.: On some knot energies involving Menger curvature. Topol. Appl. 160(13), 1507-1529 (2013). https://doi.org/10.1016/j.topol.2013.05.022

38. Strzelecki, P., von der Mosel, H.: Tangent-point self-avoidance energies for curves. J. Knot Theory Ramifications 21(5), 1250044, 28, (2012). https://doi.org/10.1142/S0218216511009960

39. Volkmann, A.: A monotonicity formula for free boundary surfaces with respect to the unit ball. Commun. Anal. Geom. 24(1), 195-221 (2016). https://doi.org/10.4310/CAG.2016.v24.n1.a7

40. von der Mosel, H.: Minimizing the elastic energy of knots. Asymptot. Anal. 18(1-2), 49-65 (1998)

41. Vorderobermeier, N.: On the regularity of critical points for O'Hara's knot energies: From smoothness to analyticity. Commun. Contemp. Math. (2020). https://doi.org/10.1142/S0219199720500455 (Paper No. 2040045)

42. Wings, A.: Stetige Differenzierbarkeit tangentenpunktartiger Knotenenergien. Bachelor thesis, RWTH Aachen University (2014)

Publisher's Note Springer Nature remains neutral with regard to jurisdictional claims in published maps and institutional affiliations. 